\title{
macrф@ufing
}

Universidade Federal de Minas Gerais

Programa de Pós-Graduação em Engenharia Elétrica

MACRO Research Group - Mecatronics, Control and Robotics

\section{WHOLE-BODY CONTROL OF A MOBILE MANIPULATOR USING FEEDBACK LINEARIZATION AND DUAL QUATERNION ALGEBRA}

Frederico Fernandes Afonso Silva

Belo Horizonte, Brazil 
Frederico Fernandes Afonso Silva

\section{WHOLE-BODY CONTROL OF A MOBILE MANIPULATOR USING FEEDBACK LINEARIZATION AND DUAL QUATERNION ALGEBRA}

Dissertation submitted to the Programa de PósGraduação em Engenharia Elétrica of Escola de Engenharia at the Universidade Federal de Minas Gerais, in partial fulfillment of the requirements for the degree of Master in Electrical Engineering.

Advisor: Bruno Vilhena Adorno 
To my family, for always encouraging and supporting me. 


\section{Acknowledgements}

Two years go fast, so much happens in between though. A master's degree has this accelerated pace and I would like to thank everyone that stayed by my side during this journey, not forgetting the ones that just arrived in my life alongside the road. Especial thanks to my parents, Flavio and Isabel, for the constant support and incentive. I would not be here without you. To my brother, Lucas, for all the laughs and company to movie marathons (specially the found footage ones).

My deepest thanks to Prof. Bruno Adorno, who has been advising me since the end of my undergraduate course. He has been a model of professionalism, ethics and management ever since. Always present in the work, in both the theoretical and practical aspects of it. I owe him a great deal of my capabilities of critical thinking, writing and work presentation. I am very grateful for all the "just five minutes" meetings, in which he would always find time in the middle of his attributions to pointing me in the right direction.

Thank you to all the UFMG's staff, for helping in my academic formation during this degree. Specially to Prof. Guilherme Raffo, Prof. Luciano Pimenta and Prof. Leonardo Tôrres, for being models of professionalism. Thank you to Vera, for never stressing with our mess in the laboratory and for always keeping things in order for us, always smiling. A especial thanks to Prof. Guilherme Raffo, for hosting the best event of the year: the annual MACRO's barbecue! He honors the traditions of the "Brazilians of the South," with the best meats and the coldest beers!

Thanks to all my friends that endured the moments of despair at the end of disciplines and papers' deadlines, without never giving up. Thank you to Gabriela (Gabi), Stella, Rafael, Diana, Fredy, Juan (Juancho), Daniel, Brenner, Arturo, Marcelo, Mariana (Mari), Letícia and Edson, for keeping the laboratory this great place to work in. Thanks also to the friends of the neighbor laboratory, Marcus, Petrus and Wendy, who never said no to a beer.

Finally, a very especial thanks to Bárbara, who, despite being in my life only since the beginning of the year, is teaching me the meaning of partnership, trust and how it is to have someone that is truly there for you. 


\section{Agradecimentos}

Dois anos passam rápido, mas tanto acontece nesse tempo. O mestrado tem um ritmo acelerado e eu gostaria de agradecer a todos aqueles que permaneceram ao meu lado durante essa jornada, sem esquecer daqueles que vieram a fazer parte da minha vida ao longo do caminho. Um agradecimento especial aos meus pais, Flavio e Isabel, por sempre me apoiar e incentivar. Eu não estaria aqui sem vocês. Ao meu irmão, Lucas, por todas as risadas e maratonas de filmes (especialmente os found footage).

Meus mais sinceros agradecimentos ao Prof. Bruno Adorno, que vem me orientando desde o final da minha graduação. Ele vem sendo um modelo de profissionalismo, ética e administração desde então. Sempre presente nos trabalhos, tanto nos aspectos teóricos quanto nos práticos. Eu lhe devo grande parte das minhas habilidades de pensamento crítico, escrita e apresentação de trabalhos. Sou muito grato por todas as reuniões de "apenas cinco minutinhos", onde ele sempre encontrava tempo em meio às suas atribuições para me apontar a direção correta.

Obrigado a todos os funcionários da UFMG, por terem ajudado na minha formação acadêmica. Um agradecimento especial aos professores Guilherme Raffo, Luciano Pimenta e Leonardo Tôrres, por serem modelos de profissionalismo. Obrigado à Vera, por nunca se estressar com as nossas bagunças no laboratório e sempre manter tudo organizado para a gente, sempre sorrindo. Um agradecimento especial ao Prof. Guilherme Raffo, por sediar o melhor evento do ano: o churrasco anual do MACRO! Ele honra as tradições dos "brasileiros do sul", com as melhores carnes e as cervejas mais geladas!

Obrigado a todos os meus amigos que suportaram os momentos de desespero de final de disciplinas e datas finais de artigos, sem nunca desistir. Obrigado Gabriela (Gabi), Stella, Rafael, Diana, Fredy, Juan (Juancho), Daniel, Brenner, Arturo, Marcelo, Mariana (Mari), Letícia e Edson, por manterem o laboratório esse ótimo lugar para se trabalhar. Obrigado também aos amigos do laboratório vizinho, Marcus, Petrus e Wendy, que nunca negaram uma cervejinha.

Finalmente, um agradecimento muito especial à Bárbara, que, apesar de estar na minha vida apenas desde o começo do ano, vem me ensinando o significado de companheirismo, confiança e como é ter alguém verdadeiramente ao seu lado. 


\section{Resumo}

Essa dissertação de mestrado apresenta o controle de corpo completo de um manipulador móvel não-holonômico utilizando controle por realimentação entrada-saída e álgebra de quatérnios duais. O controlador, cuja referência é um quatérnio dual unitário representando a pose desejada do efetuador, age como um gerador dinâmico de trajetória para o efetuador, e os sinais de entrada tanto para a base móvel não-holonômica quanto para o braço manipulador são gerados utilizando a pseudo-inversa da matriz Jacobiana de corpo completo. Para lidar com as restrições de não-holonomia, o sinal de entrada para a base móvel gerado pelo controle de corpo completo é devidamente remapeado para garantir factibilidade. Restrições nos limites das juntas, existentes na plataforma real, são tratadas como restrições na matriz Jacobiana. A estabilidade de Lyapunov para o para o sistema em malha fechada é apresentada, utilizando o método direto de Lyapunov e o teorema de Matrosov, e resultados experimentais em uma plataforma real são realizados para comparar o esquema proposto com um controlador cinemático tradicional de corpo completo. Os resultados mostram que, para uma taxa de convergência similar, o controlador não linear é capaz de gerar movimentos mais suaves, além de possuir menor esforço de controle do que o controlador linear.

Palavras-chave: Controle não linear, realimentação entrada-saída, controle cinemático, manipulador móvel não-holonômico, quatérnios duais. 


\section{Abstract}

This master thesis presents the whole-body control of a nonholonomic mobile manipulator using feedback linearization and dual quaternion algebra. The controller, whose reference is a unit dual quaternion representing the desired end-effector pose, acts as a dynamic trajectory generator for the end-effector, and input signals for both nonholonomic mobile base and manipulator arm are generated by using the pseudoinverse of the whole-body Jacobian matrix. In order to deal with the nonholonomic constraints, the input signal to the mobile base generated by the whole-body motion control is properly remapped to ensure feasibility. Joint constraints, which are present in the real platform, are treated by means of constraints in the Jacobian matrix. The Lyapunov stability for the closed-loop system is presented, utilizing Lyapunov's Direct Method and Matrosov's theorem, and experimental results on a real platform are performed in order to compare the proposed scheme to a traditional classic whole-body linear kinematic controller. The results show that, for similar convergence rate, the nonlinear controller is capable of generating smoother movements while having lower control effort than the linear controller.

Keywords: Nonlinear control, feedback linearization, kinematic control, nonholonomic mobile manipulator, dual quaternions. 


\section{Contents}

List of Figures $\quad x$

List of Tables $\quad$ xiii

Acronyms $\quad$ xiv

Notation $\quad$ Xv

1 Introduction 1

1.1 Objective and Contributions . . . . . . . . . . . . . . . . . 2

1.2 Dissertation Outline . . . . . . . . . . . . . . . . . . . 3

2 State of the Art 4

2.1 Whole-body Control . . . . . . . . . . . . . . . . . . . 4

2.2 Control of Nonholonomic Mobile Manipulators . . . . . . . . . . . 7

2.3 Nonlinear Control in Robotics _. . . . . . . . . . . . . . . . . . . 9

2.4 Joints' Constraints . . . . . . . . . . . . . . . . . . . . . . . . . 11

2.5 Chapter Conclusions . . . . . . . . . . . . . . . . . . 12

3 Mathematical Background $\quad 13$

3.1 Dual Quaternion Algebra . . . . . . . . . . . . . . . . . . 13

3.1 .1 Quaternions ....................... 13

3.1.2 Dual Quaternions . . . . . . . . . . . . . 14

3.1.2.1 Unit Dual Quaternions . . . . . . . . . . . . . . 15

3.2 Lyapunov's Theory . . . . . . . . . . . . . . . . . . . . . . . . . . 17

3.2 .1 Equilibrium Points . . . . . . . . . . . . . . . . . . 18

3.2 .2 Lyapunov's Stability . . . . . . . . . . . . . . . . . . . 18

3.2.2.1 Lyapunov's Direct Method . . . . . . . . . . . . . . . . . 19

3.2 .3 Matrosov's Theorem . . . . . . . . . . . . . . . . . . . . . 19

3.3 Chapter Conclusions . . . . . . . . . . . . . . . 20 
4 Whole-body Modeling and Control 21

4.1 Whole-body kinematics of Mobile Manipulators . . . . . . . . . . . . . 21

4.1 .1 Manipulator Kinematics . . . . . . . . . . . . . . . 22

4.1 .2 Mobile Base Kinematics . . . . . . . . . . . . . . . . 23

4.1 .3 Whole-body Kinematics . . . . . . . . . . . . . . . . . 24

4.2 Nonlinear Controller Design . . . . . . . . . . . . . . . . . 24

4.2.1 Error dynamics for the end-effector pose . . . . . . . . . 25

4.2.2 Design of the feedback linearizing control law for the end-effector pose 26

4.2.3 Whole-body controller . . . . . . . . . . . . . . . 27

4.2.4 Stability analysis of the end-effector closed-loop dynamics . . . . . 29

4.3 Classic linear kinematic control . . . . . . . . . . . . . . . . . 33

4.4 Modified Jacobian Clamping _. . . . . . . . . . . . . . . . . . . . 33

4.5 Chapter Conclusions . . . . . . . . . . . . . . . . 34

5 Experiments and Results $\quad 35$

5.1 Experimental Setup . . . . . . . . . . . . . . . . . 35

5.2 Results . . . . . . . . . . . . . . . . . 36

5.2.1 Effects of the Nonlinear Controller Gains . . . . . . . . . . . 36

5.2 .2 Comparison of Controllers . . . . . . . . . . . . . . . . 37

5.2 .3 Joints' Constraints . . . . . . . . . . . . . . . . . . . . 40

5.2.3.1 Simulations . . . . . . . . . . . . . . 40

5.2.3.2 Experiments in the Real Robot . . . . . . . . . . . 42

5.3 Chapter Conclusions . . . . . . . . . . . . . . . . . . . . 45

6 Conclusion and Future Works $\quad 48$

$\begin{array}{ll}\text { Bibliography } & 50\end{array}$ 


\section{List of Figures}

2.1 Robots used in works on whole-body control: (a) H7 (Nishiwaki et al., 2005); (b) ASIMO (courtesy of Honda); (c) Justin (courtesy of DLR) . . . . . . . 6

3.1 Three-dimensional position $\left(p_{x}, p_{y}, p_{z}\right)$ being represented by the pure quaternion $\boldsymbol{p}=\hat{\imath} p_{x}+\hat{\jmath} p_{y}+\hat{k} p_{z} . \ldots \ldots \ldots \ldots \ldots 14$

3.2 Rotation of an angle $\phi$ around a rotation axis $\boldsymbol{n}$ being represented by the

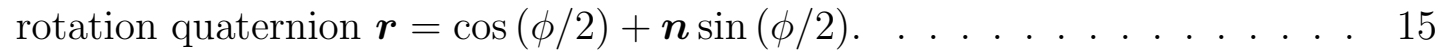

3.3 Sequence of rigid transformations represented by unit dual quaternions. . . 16

3.4 Lyapunov's concepts of stability (Slotine and Li, 1991). . . . . . . . . . . 18

3.5 Intuition behind the Matrosov's Theorem. As the function $\dot{W}_{2}$ (solid blue curve) is upper bounded by a definite negative function $-\bar{\omega}$ (red dot-dashed curve), $\lim _{t \rightarrow \infty} \dot{W}_{2}=0 . \ldots \ldots \ldots \ldots \ldots \ldots$

4.1 Mobile manipulator composed of a nonholonomic mobile base serially attached to a 5 -DOF manipulator arm. . . . . . . . . . . . .

4.2 Differential-drive mobile base. In order to deal with the nonholonomic constraint, a change in the reference coordinate system is performed. Therefore, the coordinate system is transferred from the center $(x, y)$ to a displaced point $\left(x_{d}, y_{d}\right)$.

4.3 Overall scheme for the whole-body pose control of the nonholonomic mobile manipulator

5.1 In the test scenario, the goal consisted of moving a small cube to a desired location and then dropping it into a basket. Both the robot's end-effector pose and the pose of the goal were measured in real time by using a Kinect sensor located at the ceiling and fiducial markers. . . . . . . . . . . .

5.2 Coefficients of the dual quaternion corresponding to the end-effector pose. The dotted green curve corresponds to the desired pose and in all experiments $\bar{k}_{p}=0.6$. The solid black curve corresponds to $k_{v}=1$, the blue dashed curve corresponds to $k_{v}=2$, and the red dot-dashed curve corresponds to $k_{v}=3$. 
5.3 Coefficients of the dual quaternion corresponding to the end-effector pose. The dotted green curve corresponds to the desired pose and in all experiments $k_{v}=2$. The solid black curve corresponds to $\bar{k}_{p}=0.6$, the blue dashed curve corresponds to $\bar{k}_{p}=1.4$, and the red dot-dashed curve corresponds to

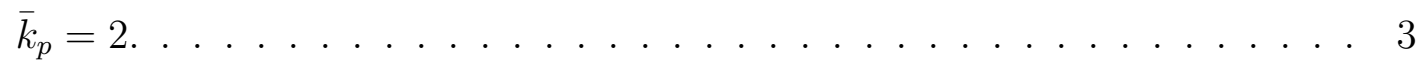

5.4 Snapshots from the experiments, where the executed trajectories from both controllers are superimposed. The semi-transparent robot (marked with a white circle) shows the motion generated by the classic controller (4.46) and the regular (not transparent) robot shows motion generated by the nonlinear controller $(4.27) . \ldots \ldots \ldots$

5.5 Coefficients of the dual quaternion error. The red dot-dashed curve corresponds to the nonlinear controller whereas the solid blue curve corresponds to the classic linear kinematic controller. . . . . . . . . . . . . . . . . .

5.6 Euclidean norm the end-effector pose error $\left(\left\|\operatorname{vec}_{8}(1-\underline{\tilde{x}})\right\|\right)$. The red dotdashed curve corresponds to the nonlinear controller whereas the solid blue curve corresponds to the classic linear kinematic controller. . . . . . . . . .

5.7 Control inputs (joints velocities). The red dot-dashed curve corresponds to the nonlinear controller whereas the solid blue curve corresponds to the classic linear kinematic controller.

5.8 Snapshots from the simulation of the Jacobian Clamping in Matlab.

5.9 Coefficients of the dual quaternion corresponding to the end-effector pose. The greyish area of the graph indicates the phase in which the robot is trying to converge to a pose that is unreachable without violating the joints' limits. The solid black curve correspond to the desired pose of the nonlinear controller. The red dot-dashed curve corresponds to the nonlinear controller without the use of Jacobian Clamping whereas the dashed blue curve corresponds to the nonlinear controller with the use of Jacobian Clamping.

5.10 Joint angles. The solid black curve correspond to the joints' limits. The red dot-dashed curve corresponds to the nonlinear controller without the use of Jacobian Clamping whereas the dashed blue curve corresponds to the nonlinear controller with the use of Jacobian Clamping. . . . . . . . . . .

5.11 Snapshots from the experiments, where the executed trajectories from both controllers are presented side by side. The images on the left show the motion generated by the nonlinear controller without the usage of the modified Jacobian Clamping and the images on the right show the motion generated by the nonlinear controller with modified Jacobian Clamping. 
5.12 Coefficients of the dual quaternion corresponding to the end-effector pose. The vertical dashed black line indicates the moment in which the motor stopped working and the experiment was interrupted. The solid black curve correspond to the desired pose of the nonlinear controller. The red dot-dashed curve corresponds to the nonlinear controller without the use of Jacobian Clamping whereas the dashed blue curve corresponds to the nonlinear controller with the use of Jacobian Clamping. . . . . . . . . . .

5.13 Joint angles. The solid black curve correspond to the joints' limits. The vertical dashed black line indicates the moment in which the motor stopped working and the experiment was interrupted. The red dot-dashed curve corresponds to the nonlinear controller without the use of Jacobian Clamping whereas the dashed blue curve corresponds to the nonlinear controller with the use of Jacobian Clamping. . . . . . . . . . . . . . . . . . . . . . . 4 


\section{List of Tables}

5.1 Control Effort. Comparison of the control effort for all controllers . . . . . 40 


\title{
Acronyms
}

\author{
ASIMO Advanced Step in Innovative MObility \\ CDTS Cooperative Dual Task-Space. \\ DLR German Aerospace Center. \\ DOF Degrees of Freedom. \\ JINT Journal of Intelligent \& Robotic Systems. \\ LARS Latin American Robotics Symposium. \\ ROS Robot Operating System. \\ SBR Brazilian Robotics Symposium. \\ UAV Unmanned Aerial Vehicle.
}




\section{Notation}

$\mathbb{H} \quad$ Set of quaternions.

$\mathcal{H} \quad$ Set of dual quaternions.

I Identity matrix.

$\hat{\imath}, \hat{\jmath}, \hat{k} \quad$ Imaginary units.

$\boldsymbol{J}^{\dagger} \quad$ Damped pseudoinverse.

$\boldsymbol{J}^{T} \quad$ Transpose of $\boldsymbol{J}$.

$\boldsymbol{J} \quad$ Jacobian matrix.

$\underline{\boldsymbol{p}} \times \underline{\boldsymbol{q}} \quad$ Cross product between dual quaternions $\underline{\boldsymbol{p}}$ and $\underline{\boldsymbol{q}}$.

$\langle\underline{\boldsymbol{p}}, \underline{\boldsymbol{q}}\rangle \quad$ Dot product between dual quaternions $\underline{\boldsymbol{p}}$ and $\underline{\boldsymbol{q}}$.

$\mathbb{R} \quad$ Set of real numbers.

Spin(3) Tridimensional spin group.

$\underline{\boldsymbol{x}}^{*} \quad$ Conjugate of dual quaternion $\underline{\boldsymbol{x}}$.

$\tilde{x} \quad$ Error related to dual quaternion $\underline{\boldsymbol{x}}$.

$\dot{x} \quad$ Time derivative of $x$.

$\varepsilon \quad$ Dual unit.

Lowercase bold letters represent column vectors or quaternions:

$$
\boldsymbol{a}=\left[\begin{array}{c}
a_{1} \\
\vdots \\
a_{n}
\end{array}\right], \quad \boldsymbol{a} \in \mathbb{R}^{n}
$$


or

$$
\boldsymbol{b}=b_{1}+\hat{\imath} b_{2}+\hat{\jmath} b_{3}+\hat{k} b_{4}, \quad \boldsymbol{b} \in \mathbb{H} .
$$

Uppercase bold letters represent matrices:

$$
\boldsymbol{A}=\left[\begin{array}{ccc}
a_{11} & \cdots & a_{1 m} \\
\vdots & \ddots & \vdots \\
a_{n 1} & \cdots & a_{n m}
\end{array}\right], \quad \boldsymbol{A} \in \mathbb{R}^{n \times m}
$$

Underlined variables represent dual numbers:

$$
\underline{a}=a_{1}+\varepsilon a_{2}, \quad \underline{\boldsymbol{a}} \in \mathcal{H},
$$

whereas

$$
\underline{\boldsymbol{h}}=\boldsymbol{h}_{1}+\varepsilon \boldsymbol{h}_{2}, \quad \underline{\boldsymbol{h}} \in \mathcal{H}
$$

is a dual quaternion. 


\section{1 \\ Introduction}

Over the last years, humanoids and mobile manipulators have attracted increasing attention in academic research, thanks to the versatility offered by such platforms. They can be used in many daily tasks such as, for example, picking up a cup (Salazar-Sangucho and Adorno, 2014), interacting with objects in shelves (Dalibard et al., 2013), entertainment (Ishida, 2004) and personal assistance (Meeussen et al., 2010; Chen et al., 2013). When interacting with humans, specially in tasks of rehabilitation, it is often undesirable (if not prejudicial) that the robot present abrupt movements (Alcocer et al., 2012; Lasota et al., 2014). In order to allow fluid and human-like movements, a strategy of whole-body motion - i.e., motions that use all available degrees of freedom (DOF) - may be used to control both humanoids and mobile manipulators.

Although humanoids are the first type of robots that may come to mind when one thinks about robots for human environments, such robots present extra challenges in the control. For instance, let us assume a simple task of picking up an object that is out of the robot's reach. In addition the proper control focused on performing the desired task, humanoids also need to perform simultaneously other tasks, such as, for example, balance and gait control. Another drawback in the usage of humanoids lies in the fact that the majority of these robots are very expensive.

On the other hand, mobile manipulators combine the dexterity of robotic arms with the mobility of mobile bases, being a versatile and low cost option to work in human environments. Another advantage of these platforms is that they do not need balance control, as the base is naturally in equilibrium. Nevertheless, one of the most common 
mobile bases is the differential mobile base, which presents the challenge of being a first order nonholonomic system (i.e., it has restrictions in the instantaneous velocities that it may execute).

Classic approaches to control mobile manipulators consists of controllers whose control effort is proportional to the error. Therefore, when the error is to large (e.g., the robot end-effector starts far from its desired pose) the robots presents abrupt movements. In order to mitigate this problem, this work explores the development of a nonlinear control technique.

In order to control the end-effector pose (i.e., position and orientation) a suitable representation should be used. Dual quaternions are one good example, since they are more compact and computationally efficient than homogeneous transformation matrices and also do not present representational singularities (Adorno, 2011). In addition, dual quaternions can be used to represent rigid motions, twists, wrenches, and several geometric primitives (e.g., Plücker lines, planes, etc.) (Adorno, 2017). Thanks to their strong algebraic properties, different robots can be modeled using the same systematic procedure (e.g., single or cooperative manipulators (Adorno et al., 2010), mobile manipulators (Adorno, 2011), and humanoids (Oliveira and Adorno, 2015; Fonseca and Adorno, 2016)), and the resultant models can be directly used with standard kinematic controllers without the need of any intermediate parameterization (Pham et al., 2010; Adorno et al., 2010; Figueredo et al., 2013). Thanks to those aforementioned advantages, dual quaternion algebra is the main mathematical tool used in this dissertation for robot modeling and control.

Real platforms often present limitations in the angles that its joints may assume. If this aspect is not considered during the controller's design and is not properly treated, it may potentially leads to instability of the system. Therefore, this work explores some existent techniques that aim to solve such problem.

\section{$1.1 \quad$ Objective and Contributions}

This work has as main objectives the design and implementation of a nonlinear kinematic controller for the whole-body control of a nonholonomic mobile manipulator. The motivation behind the usage of a nonlinear control lies in the desire to mitigate the abrupt initial movement presented by classic approaches of kinematic control (i.e. controllers whose control effort is proportional to the error), when the initial error is too large.

Partial results of this work, which uses a cascade structure composed of an outer loop with the nonlinear controller and an inner loop that explicit deals with the nonholonomic constraints of the mobile base, by using a feedback linearizing control law, were published in the 2016 XIII Latin American Robotics Symposium and IV Brazilian Robotics Symposium (LARS/SBR) (Silva and Adorno, 2016). That paper presented a comparison between the proposed strategy and the whole-body kinematic control proposed by Salazar-Sangucho 
and Adorno (2014).

The main contribution of this master's thesis is the development of a whole-body control strategy for a first order nonholonomic mobile manipulator using feedback linearization and dual quaternion algebra. Although feedback linearization has already been developed for pose control using dual quaternion algebra (Wang and Yu, 2013), those techniques combined have never been adapted and applied to first order nonholonomic mobile manipulators. In addition, this dissertation has the following novel contributions

- The nonlinear dynamical controller proposed by Wang and Yu (2013) was adapted to a nonlinear kinematic controller, extending its applicability to robots actuated on velocity or joint position (e.g. the majority of commercial mobile manipulators);

- Removal of the cascade structure proposed by Salazar-Sangucho and Adorno (2014), ensuring feasible control inputs to the nonholonomic mobile base by means of a suitable input mapping (see Section 4.2.3);

- Formal stability proof of the end-effector closed-loop dynamics, using Lyapunov's Direct Method and Matrosov's Theorem (see Section 4.2.4);

- Modification of the Jacobian Clamping (Colome and Torras, 2015), removing its limitation of permanently blocking a joint and allowing the robot to potentially regain a lost degree of freedom.

The main contributions of this dissertation were accepted with minor revisions by the Journal of Intelligent \& Robotic Systems (JINT) and the paper is currently under revision.

\subsection{Dissertation Outline}

The dissertation is structured as follows. Chapter 2 reviews important works on wholebody control, control of nonholonomic robots, nonlinear control in robotics and strategies to deal with joints' constraints. Chapter 3 presents a brief mathematical background of dual quaternion algebra, which is used for the robot modeling and controller design, Lyapunov Theory and Matrosov's Theorem, which is used in the stability proof of the end-effector closed-loop dynamics. Chapter 4 gives the modeling of the nonholonomic mobile manipulator and the whole-body control design. Chapter 5 shows the results of practical experiments, comparing the proposed structure with the classic kinematic control. Chapter 6 gives the conclusions of this work and proposals of future works. 


\section{2 \\ State of the Art}

This chapter presents a review of important works regarding whole-body control of humanoids and mobile manipulators and, also, nonlinear control in robotics. Techniques to deal with joints' constraints are also discussed in this chapter. It is divided in four sections: Section 2.1 presents a review of whole-body control, Section 2.2 reviews some control techniques of nonholonomic manipulators and Section 2.3 presents some works related with the use of nonlinear control in robotics. Section 2.4 reviews works with techniques to deal with joints' constraints.

\subsection{Whole-body Control}

Whole-body control is defined as a control technique in which all available degrees of freedom (DOF) of a robot are used at once to perform a given task. Usually, in this type of control, the goal is defined in the task space (e.g., a desired pose for the end-effector) and finding suitable joint positions to achieve it, is the task of the controller.

Nishiwaki et al. (2005) proposed a control strategy for the whole-body motion of a humanoid robot for the task of opening a crank while walking continuously. The strategy consists in first determining a desired hand position and, afterwards, deciding torso position and posture according to the position of the hand and the feet. Then, using the calculated torso and hand positions, the inverse kinematic of the arm is used to find the appropriate joint angles. Also, null space control was used to avoid joint constraints, by making the joint which is nearest to its limit farther from the limitation. The proposed control scheme 
was tested on the humanoid robot H7 (see Fig. 2.1a).

Sentis and Khatib (2006) proposed a whole-body control framework based on prioritization of tasks consisting of three hierarchical levels, where constraint-handling tasks have highest priority, followed by operational tasks, and finally posture-optimizing tasks. The tasks with lower priority are projected into the null space of the higher priority tasks. Therefore, this strategy ensures that the constraints will never be violated. The framework was tested only in simulation.

Gienger et al. (2006) presented a control algorithm that allows "displacement intervals" in task space (i.e., valid regions around a given task variable), which are exploited to satisfy one or several cost functions, similar to how redundant control techniques exploit the null space. The strategy was demonstrated through simulation and was also implemented on the humanoid robot Asimo (see Fig. 2.1b).

Nagasaka et al. (2010) proposed a whole-body cooperative force control strategy, known as Generalized Inverse Dynamics, for a mobile manipulator that can achieve different control objectives and interact with both humans and objects while performing whole-body motions. The authors also proposed a hardware configuration, called Idealized Joint Unit, to ensure accurate response to the required torque according to virtual impedance properties (inertia and viscosity). The strategy was tested on a two-armed and two-wheeled robot with 21 DOF.

Pham et al. (2010) proposed a strategy to regulate the end-effector pose of manipulator robots, which is represented by a unit dual quaternion, and uses the pseudoinverse of the robot Jacobian to generate the velocity inputs for the robot joints. By representing position and orientation in a single element, the design of the controller was simplified. The proposed scheme was tested on a six-link manipulator showing the efficiency of the method.

Dietrich et al. (2011) proposed a whole-body impedance-based controller to the DLR's mobile manipulator Justin (see Fig. 2.1c), which has two arms and a holonomic base. A synchronized behavior was achieved through the use of one single Jacobian for the whole system, except for the hands, and the approach was tested on the real platform.

Adorno (2011) presented an extension of the cooperative dual task-space (CDTS) proposed in Adorno et al. (2010) and evaluated the resultant control strategy through the simulation of a two-arm mobile manipulator for the task of pouring water. The whole-body model developed by Adorno was used in Salazar-Sangucho and Adorno (2014) to implement the whole-body control of a first order nonholonomic mobile manipulator in the task of picking and delivering a bottle of water. Park and Lee (2013) proposed a whole-body motion balance for humanoids robots based on the CDTS and evaluated the strategy by means of simulations.

Figueredo et al. (2013) proposed a $H_{\infty}$ robust controller using dual quaternion algebra to regulate the end-effector pose of manipulator robots in the presence of disturbances, 


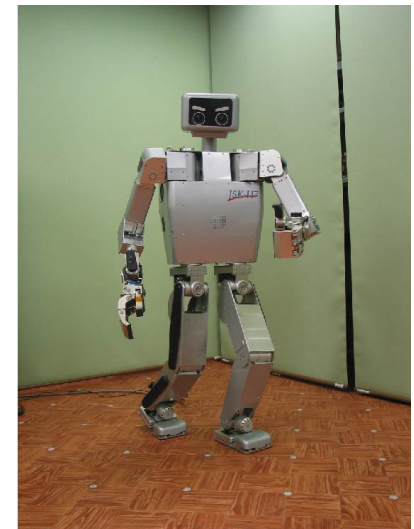

(a)

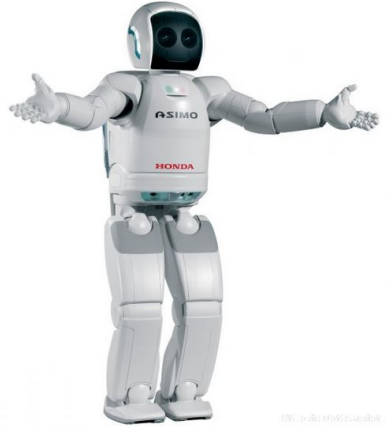

(b)

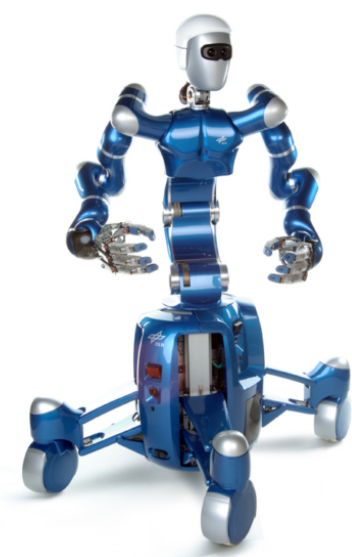

(c)

Figure 2.1: Robots used in works on whole-body control: (a) H7 (Nishiwaki et al., 2005); (b) ASIMO (courtesy of Honda); (c) Justin (courtesy of DLR).

whereas ensuring convergence. A novel error metric in dual quaternion algebra, which is invariant with respect to coordinate changes, was introduced. Simulations highlighted the effectiveness of the proposed strategy in therms of its robustness and performance.

Marinho et al. (2015) proposed an optimal controller to efficiently balance the endeffector error and its task-space velocity, using dual quaternion algebra. Departing from the invariant error definition (Figueredo et al., 2013), they derived a perturbed time-varying linear system and then proposed an optimal criterion strategy to control the error in relation to perturbations, caused by a time-varying trajectory. The strategy was tested through simulation and compared with techniques using a proportional gain controller with and without feed-forward, demonstrating the efficiency of the former in following the trajectory.

Mello et al. (2016) presented a cascade control strategy to track a desired trajectory of the end-effector, in the presence of uncertainties, of a aerial manipulator, which is composed of a robotic arm serially coupled to a quadrotor unmanned aerial vehicle (UAV). The control structure has the inner loop composed of a partial feedback linearization controller obtained from Lie derivative and the outer loop is given by a kinematic controller designed through the linear $H_{\infty}$ controller using LMIs. The whole-body kinematic model of the aerial manipulator is obtained using dual quaternion algebra.

Kussaba et al. (2017) proposed a hybrid control strategy based on unit dual quaternions to design a switching control law with hysteresis in such a way that the global asymptotic stability of the closed-loop system is guaranteed and such that the global attractivity of the stabilization pose does not exhibit chattering, a problem that is present in all discontinuous-based feedback controllers. 


\subsection{Control of Nonholonomic Mobile Manipulators}

Robotic systems are invariably subjected to a variety of constraints. A wheeled robot has, for instance, the constraint of rolling without slipping. Such constraints, called Pfaffian constraints, can be written in the form (LaValle, 2006)

$$
\phi(\boldsymbol{q}, \dot{\boldsymbol{q}}, t)=\mathbf{0},
$$

where $\boldsymbol{q}$ is the robot's configuration. If the constraints in the form of (2.1) can be integrated in such way that they can be written in the form of $\phi(\boldsymbol{q}, t)=\mathbf{0}$, they are then called holonomic constraint. On the other hand, constraints in the form of (2.1) that cannot be integrated are known as first-order nonholonomic constraints. Physically, first-order nonholonomic constraints imply that the robot is unable to achieve some instantaneous velocities. For instance, a wheeled robot, as a conventional car, is incapable of instantaneously moving in a direction normal to its wheels. It is worth noticing that this limitations in the instantaneous velocities do not prevent the robot to achieve any arbitrary pose, they simply imply that a specific set of maneuvers will be required (e.g., parallel parking maneuvers).

Li and Liu (2004) proposed the modeling and control of a mobile manipulator composed of a three-wheeled mobile platform and a four-DOF manipulator. The nonholonomic constraints of the mobile base were taken into account by means of a constraint Lagrangian formulation. To allow the end-effector to follow a given trajectory, the authors designed a model-based controller, which was proved to be asymptotically stable through Lyapunov theory. The model proposed in that paper was then used by Li and Liu (2005) and Liu and Li (2006), where controllers based on fuzzy logic were developed. In both works a fuzzy self-motion planner was used in conjunct with a robust adaptive controller and a robust adaptive neural-network controller, respectively.

De Luca et al. (2006) presented an extension of redundancy resolution techniques, originally proposed for fixed-base robotic manipulators, to mobile manipulators. The authors explicitly used the admissible mobile base velocities in the kinematic model, instead of working with its nonholonomic constraints. As for control strategy, two control schemes were implemented. The first one is based on Projected Gradients, which consists in the usage of the pseudoinverse of the Jacobian matrix and a null space projector, used to project a secondary task (e.g., joint constraints) onto the null space of the primary task. The second control scheme is based on the Reduced Gradient (De Luca and Oriolo, 1991), which performs an optimization of the objective function by directly working in the reduced space of velocity inputs that satisfy the task. Simulations performed revealed that the Reduced Gradient has superior optimization performance over the Projected Gradient method. 
Zhang et al. (2012) presented a control scheme in which a teleoperator provided the mobile manipulator's end-effector with information of desired pose. To find the solution for the redundant system, the authors suggested the modeling as a multi-objective optimization problem. As restrictions for the optimization problem, nonholonomic constraints, joint limit avoidance, singularity removal, maximum manipulability, obstacle avoidance, among others, were used. The developed controller is based on the pseudo-inversion of the robot's Jacobian and its null space projector. The effectiveness of the strategy was tested through simulation, using a mobile manipulator.

Jia et al. (2014) proposed a whole-body control strategy for a mobile manipulator composed of a mobile base and a robotic arm. The proposed scheme is composed of three levels. In the first one, a desired motion is given to the end-effector by a human operator using a spaceball. Data is sampled from the given motion and used as desired velocity for the end-effector. Finally, this desired trajectory for the end-effector is used to find a desired trajectory for the mobile base and for the manipulator. In the second level, a coordination planner receives information from the system and actively generates instantaneous input signals based on the original motion plan. The third and last level is a coordination controller that computes the joint's velocities for both the mobile base and the manipulator to achieve the instantaneous output. It is worth noticing that although the coordination controller concatenates both control signals, the mobile base and the manipulator are controlled separately and each one has its own desired trajectory (obtained in the first level). For mobile base control, the nonholonomic constraints are dealt with through a modification of the kinematic model in order to explicit entail the admissible velocities with respect to such constraints.

Salazar-Sangucho and Adorno (2014) presented a control scheme for a mobile manipulator using a cascade structure, in which the outermost level consists in a controller based on the pseudoinverse of the Jacobian matrix and the innermost loop explicitly deals with the nonholonomic constraints of the base, by means of a input-output feedback linearization. The strategy was tested in a real platform for the task of picking up and delivering a bottle of water.

Quiroz-Omana and Adorno (2016), inspired by the control structure presented by Salazar-Sangucho and Adorno (2014), proposed a control scheme using a cascade structure, in which the outermost loop was replaced by the parsimonious kinematic controller proposed by Goncalves et al. (2016), which deals with the robot motions using linear programming. Experiments on a real platform showed that this structure allows the robot to use less joints per iteration, besides allowing the integration of several constraints, such as, for example, joints' limits and low-level obstacle collision avoidance. 


\subsection{Nonlinear Control in Robotics}

Among the several existent nonlinear control strategies, one of the simplest and most used technique is the one called Feedback Linearization. This strategy consists in obtaining the error dynamics and proposing a feedback linearizing control law that will cancel the system's nonlinearities and lead it to a virtual control law. The virtual control law is then designed in order to impose the desired dynamics into the system. The general framework of a feedback linearizing control law is given by

$$
\boldsymbol{u}=\text { feedback linearization }+ \text { virtual control law. }
$$

Gilbert and Ha (1984) proposed a systematic approach for feedback system design based on a general error expression. They relied on the inverse kinematics to find the desired joint angles $\boldsymbol{q}_{d}(t)$ once a desired end-effector pose $\boldsymbol{x}_{d}(t)$ is given. Starting from the nominal model of the manipulator dynamics and using as error

$$
\widetilde{\boldsymbol{q}}=\boldsymbol{q}_{d}-\boldsymbol{q}
$$

where $\boldsymbol{q}$ are the current joint angles, the authors obtained a feedback linearizing control law that compensates the nonlinearities of the system and leads the error dynamics to a virtual control law. As virtual control law, the authors suggested the use of a simple PID controller.

Spong and Vidyasagar (1985) presented a control scheme composed of an approximate feedback control law followed by a linear compensator. The feedback linearizing controller was obtained from the error dynamics starting from the nominal model of the manipulator dynamics, while the linear compensator was designed based on the stable factorization approach (Vidyasagar, 1985) to achieve optimal tracking and disturbance rejection.

Wen et al. (1991) studied the attitude control problem of a single free-floating rigid body. The authors discussed a general controller structure in the form of

$$
\boldsymbol{u}=\text { proportional and derivative feedback }+ \text { feedforward compensation, }
$$

in which $\boldsymbol{u}$ is the control input, the proportional and derivative feedback term is used to impose the desired dynamics and the feedforward compensation is used to enhance the tracker performance. In the proposed controller, the proportional and derivative feedback terms are related to the relative attitude and to the angular velocity error, respectively. As feedforward compensation term, the authors discussed three possibilities: a model independent control law, in which no compensation is used; a compensator based on the full dynamic model with known parameters; and a compensation term with parameter adaptation. 
Following the work of Wen et al. (1991), Wang and Yu (2013) developed a pose controller for a single free-floating rigid body based on feedback linearization and dual quaternion algebra. The authors developed their control law in the form of (2.2), having terms related to the logarithm of the dual quaternion error and to the twist error as proportional and derivative feedback terms, respectively. For the feedforward compensation, the authors relied on the full dynamic model with known parameters. The results of their work were presented through simulation by using the dynamic model of a "flying brick."

de Almeida and Raffo (2015) proposed a cascade scheme with three input-output feedback linearization blocks to control a Tilt-rotor UAV carrying a suspended load. The two innermost levels control the aircraft's altitude and attitude. The third level performs path tracking while reduces the load's swing. The scheme was tested through simulation and compared with a simpler technique that uses the same controllers designed for the first and second level and replaces the third level by PID controllers, which considers the load as a disturbance. The simulations showed that the proposed scheme reduced the load swing, with an improvement of $140 \%$, at the cost of a worsening in $20 \%$ in the tracking error of the Tilt-rotor.

Rego et al. (2016) presented a leader-follower formation control problem, in which a Tiltrotor UAV performed path tracking while being followed by other Tilt-rotors UAVs. The control scheme consists of a hierarchical structure composed of a formation backstepping controller based on the Cooperative Dual Task-Space (CDTS) proposed by Adorno et al. (2010) and one linear state-feedback $\mathcal{D}$-stable $\mathcal{H}_{\infty}$ controller for each Tilt-rotor UAV, based on its linearized dynamic equations, for individual reference tracking. The proposed structure was compared through simulation with a classic technique that uses a controller based on task-space inverse dynamics for individual reference tracking, instead of the backstepping controller. The results showed that while both schemes have almost the same behavior in a disturbance-free scenario, when disturbances where added to the acceleration signals generated by the formation controller, only the backstepping controller was able to reject them.

Silva and Adorno (2016) presented an adaptation of the control strategy proposed by Wang and $\mathrm{Yu}$ (2013) that eliminated the need of obtaining the robot dynamics, allowing the application of the resultant technique to velocity-actuated robots. Therefore, it was possible to integrate the pose controller developed by Wang and Yu (2013) with the modeling from Adorno (2011) and whole-body control strategy presented by Adorno (2011); Salazar-Sangucho and Adorno (2014); Salazar-Sangucho (2014). The resulting controller uses a cascade scheme with two control loops. In the outer loop there is a nonlinear controller based on dual-quaternion feedback linearization - whose reference is a unit dual quaternion representing the desired end-effector pose - that acts as a dynamic trajectory generator and generates input signals for both nonholonomic mobile base and manipulator arm; the inner loop uses the input signals generated by the outer-loop as 
reference for an input-output linearizing controller that explicitly takes into account the nonholonomic constraints of the mobile base. The proposed strategy eliminated the abrupt initial movement due to large errors that is characteristic of kinematic controllers based on the pseudoinverse of the robot Jacobian. The proposed whole-body nonlinear controller was implemented on a mobile manipulator composed of a five-DOF robotic arm attached to a three-DOF differential mobile base. No formal proof of stability was presented in this work.

\subsection{Joints' Constraints}

Liégeois (1977) presented a control strategy using gradient projection in order to solve a secondary task in the null space of the primary. He proposed the use of the quadratic form

$$
H(\boldsymbol{q})=\frac{1}{n} \sum_{i=1}^{n}\left(\frac{q_{i}-\bar{q}}{\bar{q}-q_{i_{\max }}}\right)^{2},
$$

where $n$ is the number of joints of the robot and $\bar{q}=\left(q_{i_{\max }}+q_{i_{\min }}\right) / 2$, with $q_{i_{\max }}$ and $q_{i_{\min }}$ being the maximum and the minimum value allowed for the joint $i$, respectively, to make a manipulator draw a circle, while minimizing the deviation of the joints from its mean positions. It is worth noticing that from the value of $H(\boldsymbol{q})$ it is impossible to determine if a joint has reached its limit.

Following the work of Liégeois (1977), Zghal et al. (1990) proposed a kinematic optimization scheme for the control of manipulators with multiples degrees of redundancy, in which the gradient used was given by

$$
H(\boldsymbol{q})=\sum_{i=1}^{n} \frac{q_{i_{\max }}-q_{i_{\min }}}{\left(q_{i_{\max }}-q_{i}\right)\left(q_{i}-q_{i_{\min }}\right)} .
$$

$H(\boldsymbol{q})$ tends to infinite when approaching a joint limit and has a minimum value at the joint's mean position, therefore pushing the joints to the center of their ranges.

Chan and Dubey (1995) proposed the use of a weighting matrix to penalize joints approaching their limits. They compared their technique with strategies using gradient projection, showing that the former reduces robot self-motion while ensuring joint limit avoidance, which, on the other hand, cannot be guaranteed by the later, as joint constraints are projected into the null space of the main task.

Colome and Torras (2015) presented a strategy, known as Jacobian Clamping, to deal with joints limits that is similar to the one proposed by Chan and Dubey (1995), but 
defining the weighting matrix as a diagonal matrix $\boldsymbol{H}$, whose elements are given by

$$
h_{i}= \begin{cases}0 & , \text { if } q_{i}>q_{i_{\max }} \text { or } q_{i}<q_{i_{\min }} \\ 1 & , \text { otherwise }\end{cases}
$$

This way, any joint that violate its limit is blocked, but the joints are not pushed from their maximum (or minimum) values. If one joint is blocked, the robot loses one degree of freedom, but may continue to converge to the desired pose using the other available DOFs.

\subsection{Chapter Conclusions}

This chapter presented a review of whole-body control, control of nonholonomic manipulators, nonlinear control in robotics and strategies to deal with joints' constraints. Although the use of feedback linearization and dual quaternion algebra for pose control was already proposed in the work of Wang and Yu (2013), their goal was to control a single free-floating rigid body and they relied in the full dynamical model of it, having torques and forces as control inputs. The modification of the control structure proposed in this master thesis removes the necessity of the dynamical model of the robot, allowing the implementation of the proposed nonlinear controller in robots actuated in velocity or joint position (e.g. the majority of commercial mobile manipulators).

So far the literature has not presented works applying nonlinear control using dual quaternion algebra to first-order nonholonomic mobile manipulators. Besides, to deal with nonholonomic constraints often one relies in either including such constraints directly in the model (or in the programming of the optimization problem) or using a cascade structure. The remapping of the unfeasible control inputs into feasible ones is one of the contributions of this work.

The Jacobian Clamping as proposed by Colome and Torras (2015), although guaranteeing that the joints' limits will be respected and being simple to implement, works in such a way that, once a joint is blocked, it remains blocked forever. A modification in the definition of the matrix $\boldsymbol{H}$ proposed in this dissertation allows the joints to be potentially unblocked during the execution of the movements. This way, the robot may regain the lost degree of freedom, extending the convergence capability of the system, which was fairly limited by the original strategy.

Adapting and combining the work of Wang and Yu (2013) with the whole-body control of Salazar-Sangucho and Adorno (2014) and using the proposed modification of the Jacobian Clamping, it is possible to achieve our goals of obtaining a kinematic whole-body nonlinear control, which allows a mobile manipulator to present smooth and fluid movements, while respecting its joints' constraints. 


\section{Mathematical Background}

This chapter provides a brief revision of the principal concepts needed to understand this work. Section 3.1 gives an introduction to dual quaternion algebra, its operations and applications in robotics. Section 3.2 provides a summary of basic concepts of Lyapunov's Theory and the Matrosov's Theorem (Matrosov, 1962).

\subsection{Dual Quaternion Algebra}

This master thesis relies on dual quaternion algebra to obtain both the robot kinematics and the corresponding kinematic controllers, and thus this chapter presents some introductory concepts on dual quaternions. For a more complete account on the subject and its application to robotics, please refer to Adorno (2011); Selig (2005).

\subsubsection{Quaternions}

Quaternions were proposed by Hamilton in the 19th century (Hamilton, 1844, apud Adorno) with the purpose of extending the algebra of complex numbers, and they are elements of the set given by

$$
\mathbb{H} \triangleq\left\{h_{1}+\hat{\imath} h_{2}+\hat{\jmath} h_{3}+\hat{k} h_{4}: h_{1}, h_{2}, h_{3}, h_{4} \in \mathbb{R} \text { and } \hat{\imath}^{2}=\hat{\jmath}^{2}=\hat{k}^{2}=\hat{\imath} \hat{\jmath} \hat{k}=-1\right\} .
$$

Addition and multiplication of quaternions are analogous to their counterparts of real and complex numbers; one must only respect the properties of imaginary units $\hat{\imath}, \hat{\jmath}, \hat{k}$ given 
in (3.1). Quaternions whose real part (i.e., the element not multiplied by any imaginary unit) is equal to zero are called pure quaternions.

Pure quaternions are used to represent positions $\left(p_{x}, p_{y}, p_{z}\right)$ in the three-dimensional space by means of position quaternions

$$
\boldsymbol{p}=\hat{\imath} p_{x}+\hat{\jmath} p_{y}+\hat{k} p_{z}
$$

as shown in Fig. 3.1.

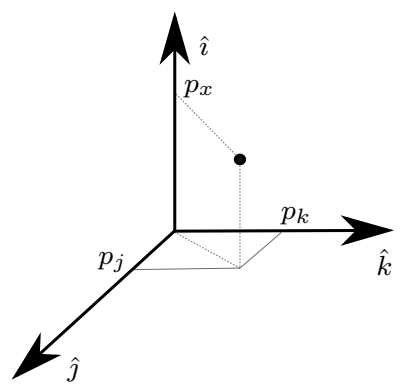

Figure 3.1: Three-dimensional position $\left(p_{x}, p_{y}, p_{z}\right)$ being represented by the pure quaternion $\boldsymbol{p}=\hat{\imath} p_{x}+\hat{\jmath} p_{y}+\hat{k} p_{z}$.

Definition 3.1 (Adorno (2017)). The norm of a quaternion $\boldsymbol{h}=h_{r}+\hat{\imath} h_{x}+\hat{\jmath} h_{y}+\hat{k} h_{z}$ is defined as

$$
\|\boldsymbol{h}\|=\sqrt{\boldsymbol{h} \boldsymbol{h}^{*}}=\sqrt{\boldsymbol{h}^{*} \boldsymbol{h}}
$$

where $\boldsymbol{h}^{*}=h_{r}-\hat{\imath} h_{x}-\hat{\jmath} h_{y}-\hat{k} h_{z}$ is the conjugate of $\boldsymbol{h}$.

Quaternions whose norm is equal to one are called unit quaternions and are used to represent rotations by means of rotation quaternions

$$
\boldsymbol{r}=\cos \left(\frac{\phi}{2}\right)+\boldsymbol{n} \sin \left(\frac{\phi}{2}\right)
$$

where $\phi$ is the rotation angle around the rotation axis $\boldsymbol{n}=\hat{\imath} n_{x}+\hat{\jmath} n_{y}+\hat{k} n_{z},\|\boldsymbol{n}\|=1$, (Selig, 2005), as illustrated in Fig. 3.2.

\subsubsection{Dual Quaternions}

Clifford extended the algebra of quaternions in 1873 giving rise to dual quaternions (Selig, 2005), which are elements of the set

$$
\mathcal{H} \triangleq\left\{\boldsymbol{h}_{P}+\varepsilon \boldsymbol{h}_{D}: \boldsymbol{h}_{P}, \boldsymbol{h}_{D} \in \mathbb{H}, \varepsilon \neq 0, \varepsilon^{2}=0\right\}
$$

Addition and multiplication of dual quaternions are analogous to their counterparts of real and complex numbers; one must only respect the properties of imaginary units $\hat{\imath}, \hat{\jmath}, \hat{k}$ and dual unit $\varepsilon$ given in (3.1) and (3.4), respectively. 


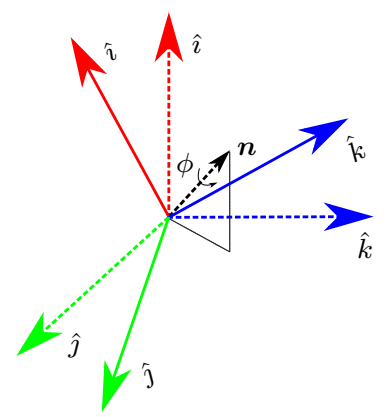

Figure 3.2: Rotation of an angle $\phi$ around a rotation axis $\boldsymbol{n}$ being represented by the rotation quaternion $\boldsymbol{r}=\cos (\phi / 2)+\boldsymbol{n} \sin (\phi / 2)$.

Definition 3.2 (Adorno (2017)). Given a dual quaternion $\underline{\boldsymbol{h}}=\boldsymbol{h}_{P}+\varepsilon \boldsymbol{h}_{D}$, the operators

$$
\begin{aligned}
& \mathcal{P}(\underline{\boldsymbol{h}}) \triangleq \boldsymbol{h}_{P}, \\
& \mathcal{D}(\underline{\boldsymbol{h}}) \triangleq \boldsymbol{h}_{D},
\end{aligned}
$$

provide the primary part and dual part of $\underline{\boldsymbol{h}}$, respectively.

Definition 3.3 (Adorno (2011)). The norm of a dual quaternion $\underline{\boldsymbol{x}}=\boldsymbol{x}_{P}+\varepsilon \boldsymbol{x}_{D}$ is given by

$$
\|\underline{\boldsymbol{x}}\|=\sqrt{{\underline{\boldsymbol{x} \boldsymbol{x}^{*}}}^{*}}=\sqrt{\underline{\boldsymbol{x}}^{*}} \underline{\boldsymbol{x}}
$$

where $\underline{\boldsymbol{x}}^{*}=\boldsymbol{x}_{P}^{*}+\varepsilon \boldsymbol{x}_{D}^{*}$. When applied to quaternions, this norm is equivalent to the Euclidean norm.

Dual quaternions whose norm is equal to one are called unit dual quaternions.

\subsubsection{Unit Dual Quaternions}

A subset of dual quaternions, the unit dual quaternions, is used to represent poses (position and orientation) in the three-dimensional space and form the group $\operatorname{Spin}(3) \ltimes \mathbb{R}^{3}$ (Selig, 2005). Unit dual quaternions can always be written as (Selig, 2005)

$$
\underline{\boldsymbol{x}}=\boldsymbol{r}+\varepsilon \frac{1}{2} \boldsymbol{p} \boldsymbol{r}
$$

where $\boldsymbol{p}$ and $\boldsymbol{r}$ are given by (3.2) and (3.3), respectively. Alternatively, (3.7) can be rewritten as

$$
\underline{\boldsymbol{x}}=\boldsymbol{r}+\varepsilon \frac{1}{2} \boldsymbol{r} \boldsymbol{p}^{b}
$$


where $\boldsymbol{p}^{b}=\operatorname{Ad}\left(\boldsymbol{r}^{*}\right) \boldsymbol{p}$ is the position with respect to the body frame, ${ }^{1}$ and $\operatorname{Ad}\left(\boldsymbol{r}^{*}\right) \boldsymbol{p} \triangleq \boldsymbol{r}^{*} \boldsymbol{p} \boldsymbol{r}$ with $\boldsymbol{r}^{*}=\cos (\phi / 2)-\boldsymbol{n} \sin (\phi / 2)$, such that $\boldsymbol{r} \boldsymbol{r}^{*}=\boldsymbol{r}^{*} \boldsymbol{r}=1$.

Unit dual quaternions as given by (3.7) or (3.8) can be used to represent a sequence of rigid transformations, wich is done by a sequence of dual quaternion multiplications. Fig. 3.3 shows a sequence of rigid transformations represented by unit dual quaternions.

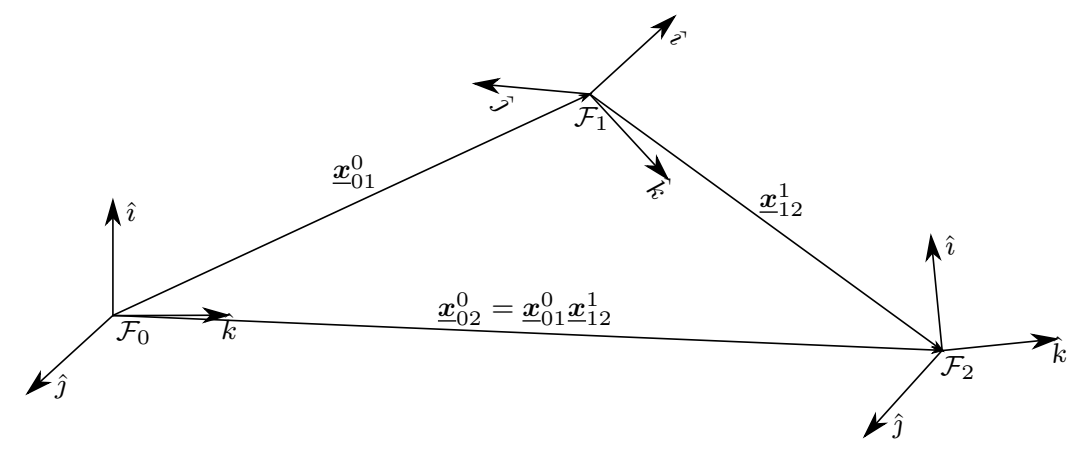

Figure 3.3: Sequence of rigid transformations represented by unit dual quaternions.

The time derivative of $\underline{\boldsymbol{x}}$ is given by (Han et al., 2008)

$$
\underline{\dot{x}}=\frac{1}{2} \underline{\boldsymbol{\xi}} \underline{\boldsymbol{x}},
$$

where $\underline{\boldsymbol{\xi}}=\boldsymbol{\omega}+\varepsilon(\dot{\boldsymbol{p}}+\boldsymbol{p} \times \boldsymbol{\omega})$ is the twist expressed in the inertial frame, with $\boldsymbol{p}$ given by (3.2) and $\boldsymbol{\omega}=\hat{\imath} \omega_{x}+\hat{\jmath} \omega_{y}+\hat{k} \omega_{z}$ is the angular velocity expressed in the inertial frame. Furthermore,

$$
\boldsymbol{p} \times \boldsymbol{\omega} \triangleq \frac{\boldsymbol{p} \boldsymbol{\omega}-\boldsymbol{\omega p}}{2}
$$

is the cross-product between pure quaternions, which is analogous to the cross product between vectors in $\mathbb{R}^{3}$ and can be verified by direct calculation (Adorno, 2017). In addition, the dot product between two pure quaternions $\boldsymbol{p}$ and $\boldsymbol{q}$ is given by

$$
\langle\boldsymbol{p}, \boldsymbol{q}\rangle \triangleq-\frac{(\boldsymbol{p} \boldsymbol{q}+\boldsymbol{q p})}{2}
$$

and can also be verified by direct calculation (Adorno, 2017).

Alternatively to (3.9),

$$
\underline{\dot{x}}=\frac{1}{2} \underline{\boldsymbol{x}}^{b}
$$

with $\underline{\boldsymbol{\xi}}^{b}=\boldsymbol{\omega}^{b}+\varepsilon\left(\dot{\boldsymbol{p}}^{b}+\boldsymbol{\omega}^{b} \times \boldsymbol{p}^{b}\right)$ being the twist expressed in the body frame (Adorno, 2017).

\footnotetext{
${ }^{1}$ The superscript indicates in which frame the quaternion or dual quaternion is represented. For instance, $\underline{x}^{b}$ represents a pose given with respect to the body frame. If no superscript is used, the global inertial frame is assumed.
} 
Definition 3.4 (Han et al. (2008)). Given a dual quaternion $\underline{\boldsymbol{x}}$ as in (3.7), its logarithm is given by

$$
\log \underline{\boldsymbol{x}}=\frac{\phi \boldsymbol{n}}{2}+\varepsilon \frac{\boldsymbol{p}}{2}
$$

with inverse mapping given by $\underline{\boldsymbol{x}}=\exp (\log \underline{\boldsymbol{x}})$ (Adorno, 2011).

Definition 3.5 (Adorno (2017)). The operator vec $: \mathcal{H} \mapsto \mathbb{R}^{8}$ gives a bijective mapping between dual quaternions and an eight-dimensional vector space. More specifically, given $\underline{\boldsymbol{x}} \in \mathcal{H}$ such that $\underline{\boldsymbol{x}}=x_{1}+\hat{\imath} x_{2}+\hat{\jmath} x_{3}+\hat{k} x_{4}+\varepsilon\left(x_{5}+\hat{\imath} x_{6}+\hat{\jmath} x_{7}+\hat{k} x_{8}\right)$, then

$$
\operatorname{vec}_{8} \underline{\boldsymbol{x}}=\left[\begin{array}{llllllll}
x_{1} & x_{2} & x_{3} & x_{4} & x_{5} & x_{6} & x_{7} & x_{8}
\end{array}\right]^{T} .
$$

Definition 3.6. The operator $\underline{\mathbf{v e c}}_{8}: \mathbb{R}^{8} \mapsto \mathcal{H}$ performs the inverse mapping given by (3.14). More specifically, given $\boldsymbol{x} \in \mathbb{R}^{8}$ such that $\boldsymbol{x}=\left[\begin{array}{llllllll}x_{1} & x_{2} & x_{3} & x_{4} & x_{5} & x_{6} & x_{7} & x_{8}\end{array}\right]^{T}$, then

$$
\underline{\mathbf{v e c}}_{8} \boldsymbol{x}=x_{1}+\hat{\imath} x_{2}+\hat{\jmath} x_{3}+\hat{k} x_{4}+\varepsilon\left(x_{5}+\hat{\imath} x_{6}+\hat{\jmath} x_{7}+\hat{k} x_{8}\right) \text {. }
$$

The vec 8 operator, together with the Hamilton operators $\stackrel{+}{\boldsymbol{H}}(\cdot)$ and $\overline{\boldsymbol{H}}(\cdot)$, are very useful to perform algebraic manipulations. More specifically, given $\underline{\boldsymbol{x}}, \underline{\boldsymbol{y}} \in \mathcal{H}$ the Hamilton operators satisfy (Adorno, 2011)

$$
\operatorname{vec}_{8}(\underline{\boldsymbol{x}} \underline{\boldsymbol{y}})=\stackrel{+}{\boldsymbol{H}}(\underline{\boldsymbol{x}}) \operatorname{vec}_{8} \underline{\boldsymbol{y}}=\overline{\boldsymbol{H}}(\underline{\boldsymbol{y}}) \operatorname{vec}_{8} \underline{\boldsymbol{x}},
$$

where

$$
\stackrel{+}{\boldsymbol{H}}(\underline{\boldsymbol{h}})=\left[\begin{array}{cc}
\stackrel{+}{\boldsymbol{H}}_{4}(\mathcal{P}(\underline{\boldsymbol{h}})) & \mathbf{0}_{4} \\
\dot{H}_{4}(\mathcal{D}(\underline{\boldsymbol{h}})) & \stackrel{+}{\boldsymbol{H}}_{4}(\mathcal{P}(\underline{\boldsymbol{h}}))
\end{array}\right], \quad \overline{\boldsymbol{H}}(\underline{\boldsymbol{h}})=\left[\begin{array}{cc}
\overline{\boldsymbol{H}}_{4}(\mathcal{P}(\underline{\boldsymbol{h}})) & \mathbf{0}_{4} \\
\overline{\boldsymbol{H}}_{4}(\mathcal{D}(\underline{\boldsymbol{h}})) & \overline{\boldsymbol{H}}_{4}(\mathcal{P}(\underline{\boldsymbol{h}}))
\end{array}\right],
$$

with

$$
\stackrel{+}{\boldsymbol{H}}_{4}(\boldsymbol{h})=\left[\begin{array}{cccc}
h_{1} & -h_{2} & -h_{3} & -h_{4} \\
h_{2} & h_{1} & -h_{4} & h_{3} \\
h_{3} & h_{4} & h_{1} & -h_{2} \\
h_{4} & -h_{3} & h_{2} & h_{1}
\end{array}\right], \quad \overline{\boldsymbol{H}}_{4}(\boldsymbol{h})=\left[\begin{array}{cccc}
h_{1} & -h_{2} & -h_{3} & -h_{4} \\
h_{2} & h_{1} & h_{4} & -h_{3} \\
h_{3} & -h_{4} & h_{1} & h_{2} \\
h_{4} & h_{3} & -h_{2} & h_{1}
\end{array}\right] .
$$

\subsection{Lyapunov's Theory}

This work relies on Lyapunov's Stability and Matrosov's Theorem (Matrosov, 1962) to develop the stability proof presented in Section 4.2.4. Therefore, some basic concepts of those theories are presented in this section. 


\subsubsection{Equilibrium Points}

If the system trajectory (i.e., the curve in state space corresponding to the system's solution) corresponds to a single point, this point is known as equilibrium point.

Definition 3.7 (Slotine and Li (1991)). A state $\boldsymbol{x}^{\prime}$ is an equilibrium state (or equilibrium point) of the system if once $\boldsymbol{x}(t)$ is equal to $\boldsymbol{x}^{\prime}$, it remains equal to $\boldsymbol{x}^{\prime}$ for all future time.

Mathematically, this means that $\boldsymbol{x}^{\prime}$ satisfies

$$
f\left(\boldsymbol{x}^{\prime}\right)=\mathbf{0}
$$

where $\dot{\boldsymbol{x}}=f(\boldsymbol{x})$.

\subsubsection{Lyapunov's Stability}

Lyapunov's concept of stability and asymptotic stability are given by the following definitions.

Definition 3.8 (Slotine and Li (1991)). The equilibrium point $\boldsymbol{x}=\mathbf{0}$ is said to be stable if, for any $R>0$, there exists $r>0$, such that if $\|\boldsymbol{x}(0)\|<r$, then $\|\boldsymbol{x}(t)\|<R$ for all $t \geq 0$. Otherwise, the equilibrium point is unstable.

In other words, if the system's trajectory starts sufficiently close to an equilibrium point and it can be kept arbitrarily close to it, then this equilibrium point is called stable. Such point is also known as marginally stable.

Definition 3.9 (Slotine and Li (1991)). An equilibrium point $\boldsymbol{x}=0$ is asymptotically stable if it is stable, and if in addition there exist some $r>0$ such that $\|\boldsymbol{x}(t)\|<r$ implies that $\boldsymbol{x}(t) \rightarrow 0$ as $t \rightarrow \infty$.

That is, if the equilibrium point is asymptotically stable, states starting close to it will converge to this equilibrium point as $t \rightarrow \infty$.

The concepts of Lyapunov's stability and asymptotic stability are illustrated in Fig. 3.4.

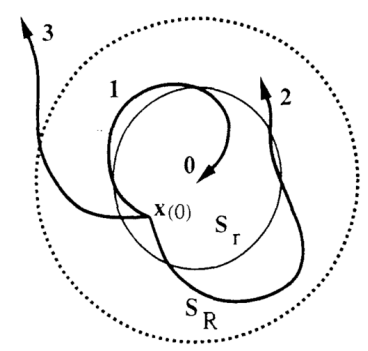

curve 1 - asymptotically stable

curve 2 - marginally stable

curve 3 - unstaule

Figure 3.4: Lyapunov's concepts of stability (Slotine and Li, 1991). 


\subsubsection{Lyapunov's Direct Method}

Lyapunov's Direct Method consists in finding a function of the states of the system and analyzing its time derivative to determine the stability of the equilibrium point at the origin. The method is stated in the Definition 3.10.

Definition 3.10 (Slotine and Li (1991)). Given a function $V(\boldsymbol{x})$ of the states of the system, such as

- $V(\boldsymbol{x})$ is differentiable

- $V(\boldsymbol{x})$ is positive definite

- $V(\boldsymbol{x}) \rightarrow \infty$ as $\|\boldsymbol{x}\| \rightarrow \infty$

if $\dot{V}(\boldsymbol{x}) \leq 0$, then the equilibrium at the origin is globally stable. If $\dot{V}(\boldsymbol{x})<0$, then the equilibrium at the origin is globally asymptotically stable.

The idea behind the method is that $V(\boldsymbol{x})$ is a "energy-like" function of the dynamic system. Therefore, by analyzing its time derivative it is possible to infer the system behavior.

\subsubsection{Matrosov's Theorem}

Originally proposed by Matrosov (1962), a simplified version of Matrosov's Theorem was presented by Astolfi and Praly (2011) and states that if $\dot{V} \leq 0$ and there exist two differentiable functions $W_{1}, W_{2}: \mathbb{R}^{n} \rightarrow \mathbb{R}$, two continuous and positive semi-definite functions $N_{1}, N_{2}: \mathbb{R}^{n} \rightarrow[0, \infty)$, a continuous function $\psi: \mathbb{R} \rightarrow \mathbb{R}$ satisfying $\psi(0)=0$, and a positive definite function $\bar{\omega}: \mathbb{R}^{n} \rightarrow \mathbb{R}$ such that

$$
\begin{aligned}
& \dot{W}_{1} \leq-N_{1} \\
& \dot{W}_{2} \leq-N_{2}+\psi\left(N_{1}\right) \\
& \bar{\omega} \leq N_{1}+N_{2}
\end{aligned}
$$

then the origin is an equilibrium point globally asymptotically stable and all bounded trajectories converge to it. Matrosov's theorem is usually used in its simplified version (Astolfi and Praly, 2011) by letting $W_{1} \triangleq V$. In addition, choosing $N_{1} \triangleq 0$ (which is clearly differentiable and positive semi-definite), then it is only necessary to choose the appropriate functions $W_{2}$ and $\bar{\omega}$ such that

$$
\dot{W}_{2} \leq-\bar{\omega}
$$

inside the set $\{\dot{V}=0\}$. 
The intuition behind Matrosov's Theorem is that, as the function $\dot{W}_{2}$ that is related to the system's states is upper bounded by the negative definite function $-\bar{\omega},{ }^{2} \lim _{t \rightarrow \infty} \dot{W}_{2}=0$ independently of its momentarily behavior in the past few instants. Fig 3.5 illustrate this idea.

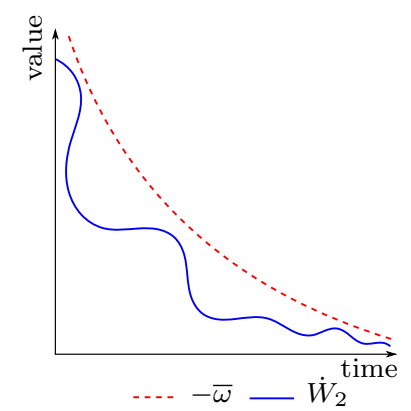

Figure 3.5: Intuition behind the Matrosov's Theorem. As the function $\dot{W}_{2}$ (solid blue curve) is upper bounded by a definite negative function $-\bar{\omega}$ (red dot-dashed curve), $\lim _{t \rightarrow \infty} \dot{W}_{2}=0$.

\subsection{Chapter Conclusions}

This chapter reviewed some fundamental mathematical concepts to this work, such as dual quaternion algebra, Lyapunov Stability and Matrosov's Theorem. Section 3.1 presented the definitions, operations, properties and applications of dual quaternions in the representation of rigid transformations. Section 3.2 reviewed the concepts of equilibrium point, Lyapunov's stability and asymptotic stability and presented the Matrosov's Theorem.

\footnotetext{
${ }^{2}$ Remember that, by definition, $\bar{\omega}$ is positive definite.
} 


\section{4 \\ Whole-body Modeling and Control}

This chapter presents the whole-body kinematic modeling of the mobile manipulator and the nonlinear control law proposed in this work. Section 4.1 shows how the whole-body kinematic model is obtained using dual quaternion algebra. Section 4.2 presents the design of the nonlinear control and the stability for the end-effector closed-loop dynamics. Section 4.3 reviews the classic kinematic control, which will be used as base of comparison to evaluate the proposed control scheme.

\subsection{Whole-body kinematics of Mobile Manipulators}

This section briefly presents the whole-body kinematics of the mobile manipulator used in this work (see Fig 4.1), starting from the robot kinematics of each subsystem (i.e. the robot manipulator and the mobile base) to the whole-body model that is used in the final controller. For more details, please refer to (Adorno, 2011).

Furthermore, it is used the mobile base iRobot Create that has three DOF but only two actuations in a differential drive configuration, which results in a first-order nonholonomic system. 


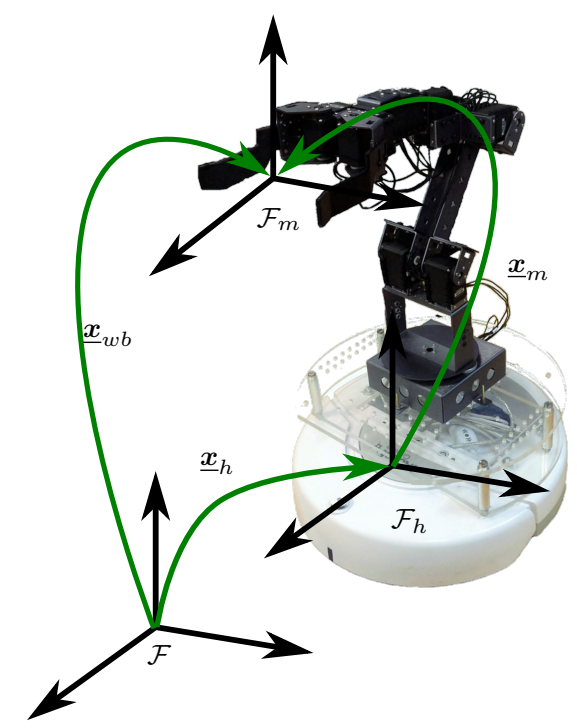

Figure 4.1: Mobile manipulator composed of a nonholonomic mobile base serially attached to a 5-DOF manipulator arm.

\subsubsection{Manipulator Kinematics}

The forward kinematics of any robot can be represented by a suitable mapping $\underline{\boldsymbol{f}}: \mathbb{R}^{n} \rightarrow$ $\operatorname{Spin}(3) \ltimes \mathbb{R}^{3}$ such that

$$
\underline{\boldsymbol{x}}=\underline{\boldsymbol{f}}(\boldsymbol{q}),
$$

where $\boldsymbol{q} \in \mathbb{R}^{n}$ is the vector of the robot configuration vector and $\underline{\boldsymbol{x}} \in \operatorname{Spin}(3) \ltimes \mathbb{R}^{3}$ is the end-effector pose (Adorno, 2011).

This way, given a mobile manipulator composed of a mobile base serially attached to a five-DOF manipulator robot, as shown in Fig. 4.1, the forward kinematics of the robot manipulator is given by

$$
\underline{\boldsymbol{x}}_{m}=\underline{\boldsymbol{f}}_{m}\left(\boldsymbol{q}_{m}\right)
$$

where $\boldsymbol{q}_{m}=\left[\begin{array}{lll}\theta_{1} & \cdots & \theta_{5}\end{array}\right]^{T}$ is the vector of manipulator joints and $\underline{\boldsymbol{x}}_{m}$ is the pose of the end-effector with respect to the manipulator base. The corresponding differential forward kinematics is

$$
\operatorname{vec}_{8} \underline{\dot{\boldsymbol{x}}}_{m}=\boldsymbol{J}_{m} \dot{\boldsymbol{q}}_{m}
$$

where $\operatorname{vec}_{8}(\cdot)$ is given by $(3.14)$ and $\boldsymbol{J}_{m} \in \mathbb{R}^{8 \times 5}$ is the manipulator Jacobian matrix that is obtained by using dual quaternion algebra (Adorno, 2011). 


\subsubsection{Mobile Base Kinematics}

When used to represent the forward kinematics of a mobile base, the dual quaternion $\underline{\boldsymbol{x}}_{h}$ that represents its pose is a function of the Cartesian coordinates $(x, y)$ and the angle $\phi$ of the base (Adorno, 2011). Therefore,

$$
\underline{\boldsymbol{x}}_{h}=\boldsymbol{r}_{h}+\frac{1}{2} \varepsilon \boldsymbol{p}_{h} \boldsymbol{r}_{h}
$$

with $\boldsymbol{p}_{h}=\hat{\imath} x+\hat{\jmath} y$ and $\boldsymbol{r}_{h}=\cos (\phi / 2)+\hat{k} \sin (\phi / 2)$.

The forward differential kinematics of the mobile base, without considering the nonholonomic constraints, is given by

$$
\operatorname{vec}_{8} \underline{\dot{\boldsymbol{x}}}_{h}=\boldsymbol{J}_{h} \dot{\boldsymbol{q}}_{h}
$$

where $\boldsymbol{q}_{h}=\left[\begin{array}{lll}x & y & \phi\end{array}\right]^{T}$ and $\boldsymbol{J}_{h} \in \mathbb{R}^{8 \times 3}$ is the holonomic Jacobian, which is also obtained algebraically (Adorno, 2011).

In order to deal with the nonholonomic constraint of the mobile base, a change in the coordinate system is performed (Siciliano et al., 2009), as illustrated in Fig. 4.2. It is easily noticeable that the point $(x, y)$ in the center of the base cannot instantaneously move in the $y$-axis. However, a displaced point $\left(x_{d}, y_{d}\right)$ does not have such constraint and this fact will be used in the end of Section 4.1 to map the control inputs generated by the whole-body controller into feasible control actions to the mobile base.

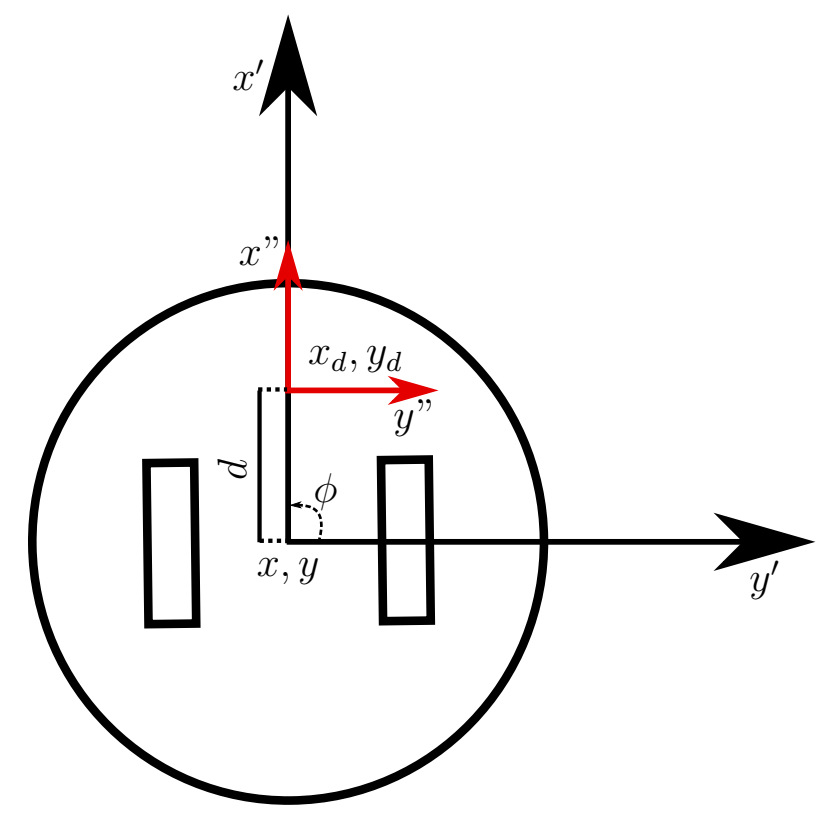

Figure 4.2: Differential-drive mobile base. In order to deal with the nonholonomic constraint, a change in the reference coordinate system is performed. Therefore, the coordinate system is transferred from the center $(x, y)$ to a displaced point $\left(x_{d}, y_{d}\right)$. 


\subsubsection{Whole-body Kinematics}

The whole-body forward kinematics is given by

$$
\underline{\boldsymbol{x}}_{w b}=\underline{\boldsymbol{x}}_{h} \underline{\boldsymbol{x}}_{m}
$$

where $\underline{\boldsymbol{x}}_{w b}$ is the end-effector pose with respect to the inertial frame and considers the coupled kinematic chain. The whole-body differential kinematics is given by (Adorno, 2011)

$$
\operatorname{vec}_{8} \underline{\dot{\boldsymbol{x}}}_{w b}=\boldsymbol{J}_{w b} \dot{\boldsymbol{q}}_{w b},
$$

where $\boldsymbol{q}_{w b}=\left[\begin{array}{ll}\boldsymbol{q}_{h}^{T} & \boldsymbol{q}_{m}^{T}\end{array}\right]^{T}$ and

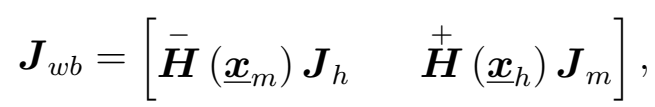

where $\stackrel{+}{\boldsymbol{H}}(\cdot)$ and $\overline{\boldsymbol{H}}(\cdot)$ are given by (3.16).

\subsection{Nonlinear Controller Design}

The overall scheme for the whole-body pose control of the mobile manipulator is shown in Fig. 4.3. The reference for the nonlinear controller is a unit dual quaternion representing the desired end-effector pose. The resultant input signal from the nonlinear controller is split into two input signals, one for the manipulator and other for the nonholonomic mobile base. The control signal generated for the latter is properly mapped into feasible inputs to deal with the nonholonomic constraints of the differential mobile base.

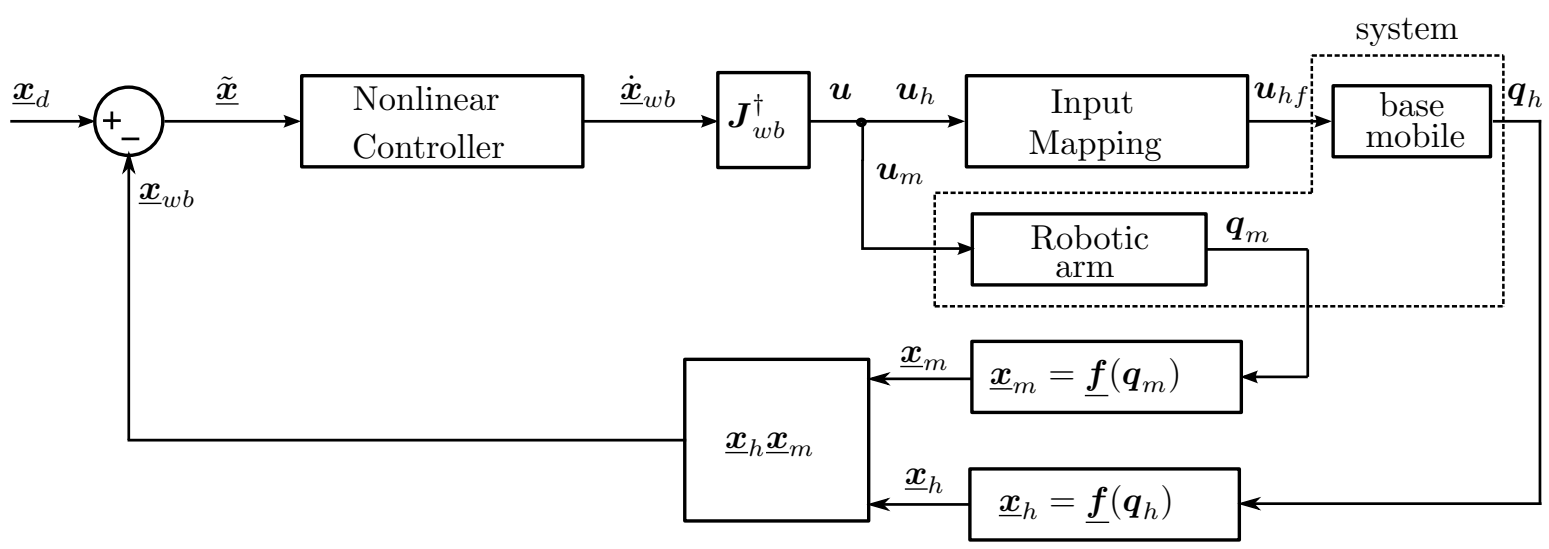

Figure 4.3: Overall scheme for the whole-body pose control of the nonholonomic mobile manipulator 


\subsubsection{Error dynamics for the end-effector pose}

In order to design the nonlinear controller, first a suitable error in unit dual quaternion space must be defined, such as

$$
\begin{aligned}
\underline{\tilde{\boldsymbol{x}}} & \triangleq \underline{\boldsymbol{x}}_{d}^{*} \underline{\boldsymbol{x}}_{w b} \\
& =\tilde{\boldsymbol{r}}+\frac{1}{2} \tilde{\boldsymbol{r}} \tilde{\boldsymbol{p}}^{b},
\end{aligned}
$$

where $\underline{\boldsymbol{x}}_{w b}=\boldsymbol{r}_{w b}+\varepsilon(1 / 2) \boldsymbol{r}_{w b} \boldsymbol{p}_{w b}^{b}$ and $\underline{\boldsymbol{x}}_{d}=\boldsymbol{r}_{d}+\varepsilon(1 / 2) \boldsymbol{r}_{d} \boldsymbol{p}_{d}^{b}$ are the current and desired end-effector pose, respectively, and $\tilde{\boldsymbol{r}}=\cos (\tilde{\theta} / 2)+\boldsymbol{n} \sin (\tilde{\theta} / 2)$.

It is important to note that $\underline{\boldsymbol{x}}_{w b} \rightarrow \underline{\boldsymbol{x}}_{d}$ implies $\underline{\tilde{\boldsymbol{x}}} \rightarrow 1$, which in turn implies $\tilde{\boldsymbol{p}}^{b} \rightarrow 0$ and $\tilde{\boldsymbol{r}} \rightarrow 1$. Last, $\tilde{\boldsymbol{r}} \rightarrow 1$ implies $\tilde{\theta} \rightarrow 0$.

The time derivative of $\underline{\boldsymbol{x}}$ is given by

$$
\begin{aligned}
\underline{\dot{\boldsymbol{x}}} & =\underline{\boldsymbol{x}}_{d}^{*} \underline{\boldsymbol{x}}_{w b}+\underline{\boldsymbol{x}}_{d}^{*} \underline{\boldsymbol{x}}_{w b} \\
& =\left(\frac{1}{2} \underline{\boldsymbol{x}}_{d} \underline{\boldsymbol{\xi}}_{d}^{b}\right)^{*} \underline{\boldsymbol{x}}_{w b}+\underline{\boldsymbol{x}}_{d}^{*}\left(\frac{1}{2} \underline{\boldsymbol{x}}_{w b} \underline{\boldsymbol{\xi}}_{w b}^{b}\right) \\
& =-\frac{1}{2} \underline{\boldsymbol{\xi}}_{d}^{b} \underline{\boldsymbol{x}}_{d}^{*} \underline{\boldsymbol{x}}_{w b}+\frac{1}{2} \underline{\boldsymbol{x}}_{d}^{*} \underline{\boldsymbol{x}}_{w b} \underline{\boldsymbol{\xi}}_{w b}^{b} \\
& =-\frac{1}{2} \underline{\boldsymbol{\xi}}_{d}^{b} \underline{\tilde{\boldsymbol{x}}}+\frac{1}{2} \underline{\tilde{\boldsymbol{x}}}_{w b}^{b} \\
& =\frac{1}{2} \underline{\tilde{\boldsymbol{x}}}_{w}\left(\underline{\boldsymbol{\xi}}_{w b}^{b}-\operatorname{Ad}\left(\underline{\tilde{\boldsymbol{x}}}^{*}\right) \underline{\boldsymbol{\xi}}_{d}^{b}\right),
\end{aligned}
$$

where $\operatorname{Ad}\left(\underline{\tilde{\boldsymbol{x}}}^{*}\right) \underline{\boldsymbol{\xi}}_{d}^{b} \triangleq \underline{\tilde{\boldsymbol{x}}}^{*} \underline{\boldsymbol{\xi}}_{d}^{b} \underline{\tilde{\boldsymbol{x}}}$, with $\underline{\boldsymbol{x}}^{*}=\boldsymbol{x}_{P}^{*}+\varepsilon \boldsymbol{x}_{D}^{*}$.

Defining

$$
\underline{\tilde{\boldsymbol{\xi}}}^{b}=\underline{\boldsymbol{\xi}}_{w b}^{b}-\operatorname{Ad}\left(\underline{\tilde{\boldsymbol{x}}}^{*}\right) \underline{\boldsymbol{\xi}}_{d}^{b}
$$

the time derivative of the dual quaternion error is given by

$$
\underline{\dot{\tilde{x}}}=\frac{1}{2} \underline{\tilde{\boldsymbol{x}}} \underline{\tilde{\boldsymbol{\xi}}}^{b} .
$$




\subsubsection{Design of the feedback linearizing control law for the end- effector pose}

The time derivative of $\underline{\tilde{\xi}}^{b}$ is given by ${ }^{12}$

$$
\begin{aligned}
& \underline{\tilde{\boldsymbol{\xi}}}^{b}=\underline{\boldsymbol{\xi}}_{w b}^{b}-\left(\underline{\tilde{\tilde{\boldsymbol{x}}}}^{*} \underline{\boldsymbol{\xi}}_{d}^{b} \underline{\tilde{\boldsymbol{x}}}+\underline{\tilde{\boldsymbol{x}}}^{*} \underline{\boldsymbol{\xi}}_{d}^{b} \underline{\tilde{\boldsymbol{x}}}+\underline{\tilde{\boldsymbol{x}}}^{*} \underline{\boldsymbol{\xi}}_{d}^{b} \dot{\tilde{\boldsymbol{x}}}\right) \\
& =\underline{\boldsymbol{\xi}}_{w b}^{b}-\left(-\frac{1}{2} \underline{\tilde{\boldsymbol{\xi}}}^{b} \underline{\tilde{\boldsymbol{x}}}^{*} \underline{\boldsymbol{\xi}}_{d}^{b} \underline{\tilde{\boldsymbol{x}}}+\underline{\tilde{\boldsymbol{x}}}^{*} \underline{\boldsymbol{\xi}}_{d}^{b} \underline{\tilde{\boldsymbol{x}}}+\frac{1}{2} \underline{\tilde{\boldsymbol{x}}}^{*} \underline{\boldsymbol{\xi}}_{d}^{b} \underline{\tilde{\boldsymbol{x}}}^{\tilde{\boldsymbol{\xi}}^{b}}\right) \\
& =\underline{\dot{\boldsymbol{\xi}}}_{w b}^{b}-\left(\operatorname{Ad}\left(\underline{\tilde{\boldsymbol{x}}}^{*}\right) \underline{\boldsymbol{\xi}}_{d}^{b}+\frac{\left(\operatorname{Ad}\left(\underline{\tilde{\boldsymbol{x}}}^{*}\right) \underline{\boldsymbol{\xi}}_{d}^{b}\right) \underline{\tilde{\boldsymbol{\xi}}}^{b}-\underline{\tilde{\boldsymbol{\xi}}}^{b}\left(\operatorname{Ad}\left(\underline{\tilde{\boldsymbol{x}}}^{*}\right) \underline{\boldsymbol{\xi}}_{d}^{b}\right)}{2}\right) \\
& =\underline{\dot{\boldsymbol{\xi}}}_{w b}^{b}-\operatorname{Ad}\left(\underline{\tilde{\boldsymbol{x}}}^{*}\right) \underline{\boldsymbol{\xi}}_{d}^{b}-\left(\operatorname{Ad}\left(\underline{\tilde{\boldsymbol{x}}}^{*}\right) \underline{\boldsymbol{\xi}}_{d}^{b}\right) \times \underline{\tilde{\boldsymbol{\xi}}}^{b},
\end{aligned}
$$

where the cross-product between two pure dual quaternions ${ }^{3}$ is analogous to the crossproduct (3.10). More specifically, given two pure dual quaternions $\underline{\boldsymbol{a}}$ and $\underline{\boldsymbol{b}}$,

$$
\underline{a} \times \underline{b} \triangleq \frac{a b}{-b} \underline{\boldsymbol{a}}
$$

Considering (4.12) and defining $\underline{\dot{\xi}}_{w b}^{b}$ as the feedback linearizing control input; that is,

$$
\underline{\boldsymbol{U}} \triangleq \underline{\dot{\boldsymbol{\xi}}}_{w b}^{b}
$$

it is possible to impose into the system the desired dynamics. The feedback linearizing control law proposed in (Wang and Yu, 2013) is adapted to obtain ${ }^{4}$

$$
\underline{\boldsymbol{U}}=-k_{p} \log \underline{\tilde{\boldsymbol{x}}}-k_{v} \underline{\tilde{\boldsymbol{\xi}}}^{b}+\operatorname{Ad}\left(\underline{\tilde{\boldsymbol{x}}}^{*}\right) \underline{\boldsymbol{\xi}}_{d}^{b}+\left(\operatorname{Ad}\left(\underline{\tilde{\boldsymbol{x}}}^{*}\right) \underline{\boldsymbol{\xi}}_{d}^{b}\right) \times \underline{\tilde{\boldsymbol{\xi}}}^{b},
$$

where $k_{p}$ and $k_{v}$ are real positive gains and $\log (\cdot)$ is given by (3.13).

Substituting (4.14) into (4.12), we obtain the closed-loop error dynamics

$$
\underline{\dot{\boldsymbol{\xi}}}^{b}=-k_{p} \log \underline{\tilde{\boldsymbol{x}}}-k_{v} \underline{\tilde{\boldsymbol{\xi}}}^{b}
$$

As in this work we are dealing with the first order differential kinematics (4.7), the

\footnotetext{
${ }^{1}$ Here it is assumed that the robot is actuated in accelerations. Therefore, $\dot{\tilde{\boldsymbol{\xi}}}^{b}$ needs to be calculated.

${ }^{2}$ It is important to highlight that in the case of a regulation problem-i.e., $\underline{\dot{x}}_{d}(t)=0$-we have $\underline{\boldsymbol{\xi}}_{d}^{b}=\dot{\boldsymbol{\xi}}_{d}^{b}=0$, therefore $\underline{\dot{\boldsymbol{\xi}}}^{b}=\underline{\dot{\boldsymbol{\xi}}}_{w b}^{b}$.

${ }^{3}$ A dual quaternion $\underline{\boldsymbol{h}}=\underline{\boldsymbol{h}}_{P}+\varepsilon \boldsymbol{h}_{D}$ is pure if both $\boldsymbol{h}_{P}$ and $\boldsymbol{h}_{D}$ are pure quaternions.

${ }^{4}$ Since Wang and Yu Wang and Yu (2013) followed a different path to deduce both the body twist and the error twist, they obtained an equation equivalent to (4.12), but with different nonlinear terms that must be linearized.
} 
control input will be given by

$$
\underline{\boldsymbol{u}}_{\xi} \triangleq \underline{\boldsymbol{\xi}}_{w b}^{b}
$$

rather than by its time derivative (4.13). Therefore, the current value of $\underline{\boldsymbol{\xi}}_{w b}^{b}$ can be obtained from (4.7) and (3.12) as

$$
\underline{\dot{\boldsymbol{x}}}_{w b}=\underline{\mathbf{v e c}}_{8}\left(\boldsymbol{J}_{w b} \dot{\boldsymbol{q}}_{w b}\right)=\frac{1}{2} \underline{\boldsymbol{x}}_{w b} \underline{\boldsymbol{\xi}}_{w b}^{b} \Longrightarrow \underline{\boldsymbol{u}}_{\xi}=2 \underline{\boldsymbol{x}}_{w b}^{*} \underline{\mathbf{v e c}}_{8}\left(\boldsymbol{J}_{w b} \dot{\boldsymbol{q}}_{w b}\right),
$$

where $\underline{\mathbf{v e c}}_{8}(\cdot)$ is given by $(3.15)$.

In order to be implemented in the real system, (4.17) must be discretized. By using the Euler method, and then using (4.13), (4.14), and (4.17), the (body) twist input is obtained

$$
\underline{\boldsymbol{u}}_{\xi}=T \underline{\boldsymbol{U}}+2 \underline{\boldsymbol{x}}_{w b}^{*} \underline{\mathbf{v e c}}_{8}\left(\boldsymbol{J}_{w b} \dot{\boldsymbol{q}}_{w b}\right),
$$

where $T$ is the integration step.

\subsubsection{Whole-body controller}

Eq. (4.18) provides the control input for the end-effector, but the actual robot inputs are the joint velocities; that is, $\boldsymbol{u} \triangleq \dot{\boldsymbol{q}}_{w b}{ }^{5}$ This way, using (4.7) we obtain the whole-body control inputs

$$
\boldsymbol{u}=\boldsymbol{J}_{w b}^{\dagger} \operatorname{vec}_{8}\left(\frac{1}{2} \underline{\boldsymbol{x}}_{w b} \underline{\boldsymbol{u}}_{\xi}\right)
$$

where the matrix $\boldsymbol{J}_{w b}^{\dagger} \triangleq \boldsymbol{J}_{w b}^{T}\left(\boldsymbol{J}_{w b} \boldsymbol{J}_{w b}^{T}+\lambda^{2} \boldsymbol{I}\right)^{-1}$ is the damped pseudoinverse of $\boldsymbol{J}_{w b}$ and $\lambda$ is the damping factor (Chiaverini, 1997).

The whole-body control input (4.19) is partitioned into two parts, that is,

$$
\boldsymbol{u}=\left[\begin{array}{c}
\boldsymbol{u}_{h} \\
\boldsymbol{u}_{m}
\end{array}\right]
$$

where $\boldsymbol{u}_{h}=\left[\begin{array}{lll}\dot{x} & \dot{y} & \dot{\phi}\end{array}\right]^{T}$ is the control input for the the mobile base and $\boldsymbol{u}_{m}=\left[\begin{array}{lll}\dot{\theta}_{1} & \cdots & \dot{\theta}_{5}\end{array}\right]^{T}$ is the control input for the manipulator. It is important to note that, as explained in Section 4.1.2, the inputs $\boldsymbol{u}_{h}$ may be unfeasible due to the nonholonomic constraint of the mobile base. This problem is solved in the sequel.

The relation between the time derivative of the mobile base coordinates $(x, y, \phi)$ and

\footnotetext{
${ }^{5}$ Since the kinematic model (4.7) is used, a single integrator dynamics is assumed.
} 
its linear velocity $v$ and angular velocity $\omega$ is given by

$$
\left[\begin{array}{c}
\dot{x} \\
\dot{y} \\
\dot{\phi}
\end{array}\right]=\left[\begin{array}{cc}
\cos \phi & 0 \\
\sin \phi & 0 \\
0 & 1
\end{array}\right]\left[\begin{array}{l}
v \\
\omega
\end{array}\right]
$$

The coordinates $\left(x_{d}, y_{d}\right)$, illustrated in Fig. 4.2 , are given by

$$
\begin{aligned}
& x_{d}=x+d \cos \phi \\
& y_{d}=y+d \sin \phi,
\end{aligned}
$$

with time derivative given by

$$
\left[\begin{array}{c}
\dot{x}_{d} \\
\dot{y}_{d}
\end{array}\right]=\underbrace{\left[\begin{array}{ccc}
1 & 0 & -d \sin \phi \\
0 & 1 & d \cos (\phi)
\end{array}\right]}_{\boldsymbol{B}}\left[\begin{array}{c}
\dot{x} \\
\dot{y} \\
\dot{\phi}
\end{array}\right] .
$$

Replacing (4.21) in (4.23) yields

$$
\left[\begin{array}{l}
\dot{x}_{d} \\
\dot{y}_{d}
\end{array}\right]=\underbrace{\left[\begin{array}{cc}
\cos (\phi) & -d \sin (\phi) \\
\sin (\phi) & d \cos (\phi)
\end{array}\right]}_{C}\left[\begin{array}{c}
v \\
\omega
\end{array}\right],
$$

therefore $^{6}$

$$
\begin{aligned}
{\left[\begin{array}{c}
v \\
\omega
\end{array}\right] } & =\boldsymbol{C}^{-1}\left[\begin{array}{l}
\dot{x}_{d} \\
\dot{y}_{d}
\end{array}\right] \\
& =\boldsymbol{C}^{-1} \boldsymbol{B}\left[\begin{array}{c}
\dot{x} \\
\dot{y} \\
\dot{\phi}
\end{array}\right]
\end{aligned}
$$

The relation between the velocities $\omega_{r}$ and $\omega_{l}$ of the right and left wheels, respectively, and the mobile base's linear and angular velocities is given by (Siciliano et al., 2009)

$$
\left[\begin{array}{c}
\omega_{r} \\
\omega_{l}
\end{array}\right]=\underbrace{\frac{1}{r}\left[\begin{array}{cc}
1 & b \\
1 & -b
\end{array}\right]}_{\boldsymbol{D}}\left[\begin{array}{l}
v \\
\omega
\end{array}\right],
$$

where $r$ is the radius of the wheels and $b$ is the half of the distance distance between them.

\footnotetext{
${ }^{6}$ It is straightforward to verify that $\operatorname{det} \boldsymbol{C}=d$, thus $\boldsymbol{C}^{-1}$ always exists $\forall d \neq 0$.
} 
Substituting (4.24) in (4.25) yields the feasible control inputs $\boldsymbol{u}_{h f}$ for the mobile base:

$$
\boldsymbol{u}_{h f} \triangleq\left[\begin{array}{c}
\omega_{r} \\
\omega_{l}
\end{array}\right]=\boldsymbol{D} \boldsymbol{C}^{-1} \boldsymbol{B}\left[\begin{array}{c}
\dot{x} \\
\dot{y} \\
\dot{\phi}
\end{array}\right]
$$

Finally, considering (4.19), (4.20), and (4.26) the feasible whole-body control input is obtained

$$
\boldsymbol{u}_{f}=\left[\begin{array}{l}
\boldsymbol{u}_{h f} \\
\boldsymbol{u}_{m}
\end{array}\right]=\underbrace{\left[\begin{array}{cc}
\boldsymbol{D} \boldsymbol{C}^{-1} \boldsymbol{B} & \mathbf{0} \\
\mathbf{0} & \boldsymbol{I}
\end{array}\right]}_{\boldsymbol{F}} \boldsymbol{u} \Longrightarrow \boldsymbol{u}_{f}=\boldsymbol{F} \boldsymbol{J}_{w b}^{\dagger} \operatorname{vec}_{8}\left(\frac{1}{2} \underline{\boldsymbol{x}}_{w b} \underline{\boldsymbol{u}}_{\xi}\right) .
$$

\subsubsection{Stability analysis of the end-effector closed-loop dynamics}

In this section is proved the stability for the end-effector closed-loop dynamics under control law (4.14).

Theorem 4.1. Defining

$$
\begin{aligned}
V_{\xi} & =\left\|\mathcal{P}\left(\underline{\tilde{\boldsymbol{\xi}}}^{b}\right)\right\|^{2}+\left\|\mathcal{D}\left(\underline{\tilde{\boldsymbol{\xi}}}^{b}\right)\right\|^{2} \\
& =\mathcal{P}\left(\underline{\tilde{\boldsymbol{\xi}}}^{b}\right) \mathcal{P}\left(\underline{\tilde{\boldsymbol{\xi}}}^{b}\right)^{*}+\mathcal{D}\left(\underline{\tilde{\boldsymbol{\xi}}}^{b}\right) \mathcal{D}\left(\underline{\tilde{\boldsymbol{\xi}}}^{b}\right)^{*}
\end{aligned}
$$

and

$$
\begin{aligned}
V_{\text {log }} & =\bar{k}_{p}\|\mathcal{P}(\log \underline{\tilde{\boldsymbol{x}}})\|^{2}+\bar{k}_{p}\|\mathcal{D}(\log \underline{\tilde{\boldsymbol{x}}})\|^{2} \\
& =\bar{k}_{p} \mathcal{P}(\log \underline{\tilde{\boldsymbol{x}}}) \mathcal{P}(\log \underline{\tilde{\boldsymbol{x}}})^{*}+\bar{k}_{p} \mathcal{D}(\log \underline{\tilde{\boldsymbol{x}}}) \mathcal{D}(\log \underline{\tilde{\boldsymbol{x}}})^{*},
\end{aligned}
$$

where $\|\cdot\|$ is the norm as defined in (3.6), the operators $\mathcal{P}(\cdot)$ and $\mathcal{D}(\cdot)$ are defined in (3.5), and $\bar{k}_{p}$ is a positive real number, ${ }^{7}$ and considering the candidate Lyapunov function ${ }^{8}$

$$
V=V_{\xi}+V_{\log }
$$

the origin $\underline{\tilde{\boldsymbol{x}}}=1$ is a stable equilibrium point for the closed-loop error dynamics given by (4.11) under control law given by (4.14).

\footnotetext{
${ }^{7}$ The gain $\bar{k}_{p}$ was introduced in order to provide more freedom into the controller's design and adjust, as will be clearer in (4.44).

${ }^{8}$ It is clear that $V \geq 0$ and $V=0 \Longleftrightarrow \underline{\tilde{\xi}}^{b}=\log \underline{\tilde{x}}=0$, because the quaternion norm is equivalent to the Euclidean norm.
} 
Proof. The time derivative of $V$ is given by

$$
\dot{V}=\dot{V}_{\xi}+\dot{V}_{\log }
$$

where

$$
\begin{aligned}
\dot{V}_{\log }=\bar{k}_{p} \frac{d}{d t} \mathcal{P}(\log \underline{\tilde{\boldsymbol{x}}}) \mathcal{P}(\log \underline{\tilde{\boldsymbol{x}}})^{*}+\bar{k}_{p} \mathcal{P}(\log \underline{\tilde{\boldsymbol{x}}}) \frac{d}{d t} \mathcal{P}(\log \underline{\tilde{\boldsymbol{x}}})^{*}+ \\
\bar{k}_{p} \frac{d}{d t} \mathcal{D}(\log \underline{\tilde{\boldsymbol{x}}}) \mathcal{D}(\log \underline{\tilde{\boldsymbol{x}}})^{*}+\bar{k}_{p} \mathcal{D}(\log \underline{\tilde{\boldsymbol{x}}}) \frac{d}{d t} \mathcal{D}(\log \underline{\tilde{\boldsymbol{x}}})^{*},
\end{aligned}
$$

which, using the definition of dot product (3.11), yields

$$
\dot{V}_{\log }=2 \bar{k}_{p} A_{1}
$$

where

$$
A_{1} \triangleq\left\langle\mathcal{P}(\log \underline{\tilde{\boldsymbol{x}}}), \frac{d}{d t} \mathcal{P}(\log \underline{\tilde{\boldsymbol{x}}})\right\rangle+\left\langle\mathcal{D}(\log \underline{\tilde{\boldsymbol{x}}}), \frac{d}{d t} \mathcal{D}(\log \underline{\tilde{\boldsymbol{x}}})\right\rangle
$$

and

$$
\begin{aligned}
\dot{V}_{\xi}=\frac{d}{d t} \mathcal{P}\left(\underline{\tilde{\boldsymbol{\xi}}}^{b}\right) \mathcal{P}\left(\underline{\tilde{\boldsymbol{\xi}}}^{b}\right)^{*}+\mathcal{P}\left(\underline{\tilde{\boldsymbol{\xi}}}^{b}\right) & \frac{d}{d t} \mathcal{P}\left(\underline{\tilde{\boldsymbol{\xi}}}^{b}\right)^{*} \\
& +\frac{d}{d t} \mathcal{D}\left(\underline{\tilde{\boldsymbol{\xi}}}^{b}\right) \mathcal{D}\left(\underline{\tilde{\boldsymbol{\xi}}}^{b}\right)^{*}+\mathcal{D}\left(\underline{\tilde{\xi}}^{b}\right) \frac{d}{d t} \mathcal{D}\left(\underline{\tilde{\boldsymbol{\xi}}}^{b}\right)^{*} .
\end{aligned}
$$

In addition, since

$$
\underline{\dot{\boldsymbol{\xi}}}^{b}=\frac{d}{d t} \mathcal{P}\left(\underline{\tilde{\boldsymbol{\xi}}}^{b}\right)+\varepsilon \frac{d}{d t} \mathcal{D}\left(\underline{\tilde{\xi}}^{b}\right)
$$

and the closed-loop error dynamics under feedback linearizing control law (4.14) is given by $(4.15)$, thus

$$
\begin{aligned}
\frac{d}{d t} \mathcal{P}\left(\underline{\tilde{\boldsymbol{\xi}}}^{b}\right)+\varepsilon \frac{d}{d t} \mathcal{D}\left(\underline{\tilde{\boldsymbol{\xi}}}^{b}\right) & =-k_{p} \log \underline{\tilde{\boldsymbol{x}}}-k_{v} \underline{\tilde{\boldsymbol{\xi}}}^{b} \\
& =-k_{p} \mathcal{P}(\log \underline{\tilde{\boldsymbol{x}}})-\varepsilon k_{p} \mathcal{D}(\log \underline{\tilde{\boldsymbol{x}}})-k_{v} \mathcal{P}\left(\underline{\tilde{\boldsymbol{\xi}}}^{b}\right)-\varepsilon k_{v} \mathcal{D}\left(\underline{\tilde{\boldsymbol{\xi}}}^{b}\right) .
\end{aligned}
$$

Therefore, ${ }^{9}$

$$
\begin{aligned}
& \frac{d}{d t} \mathcal{P}\left(\underline{\tilde{\boldsymbol{\xi}}}^{b}\right)=-k_{p} \mathcal{P}(\log \underline{\tilde{\boldsymbol{x}}})-k_{v} \mathcal{P}\left(\underline{\tilde{\boldsymbol{\xi}}}^{b}\right), \\
& \frac{d}{d t} \mathcal{D}\left(\underline{\tilde{\boldsymbol{\xi}}}^{b}\right)=-k_{p} \mathcal{D}(\log \underline{\tilde{\boldsymbol{x}}})-k_{v} \mathcal{D}\left(\underline{\tilde{\boldsymbol{\xi}}}^{b}\right) .
\end{aligned}
$$

${ }^{9}$ Since $\underline{\tilde{\xi}}^{b}$ is a pure dual quaternion, $\frac{d}{d t} \mathcal{P}\left(\underline{\tilde{\xi}}^{b}\right)^{*}=-\frac{d}{d t} \mathcal{P}\left(\underline{\tilde{\xi}}^{b}\right)$. 
Substituting (4.35) in (4.34) yields ${ }^{10}$

$$
\begin{aligned}
\dot{V}_{\xi}=-k_{p} \mathcal{P}(\log \underline{\tilde{\boldsymbol{x}}}) \mathcal{P} & \left(\underline{\tilde{\boldsymbol{\xi}}}^{b}\right)^{*}-k_{v} \mathcal{P}\left(\underline{\tilde{\boldsymbol{\xi}}}^{b}\right) \mathcal{P}\left(\underline{\tilde{\boldsymbol{\xi}}}^{b}\right)^{*} \\
& \quad-k_{p} \mathcal{P}\left(\underline{\tilde{\boldsymbol{\xi}}}^{b}\right) \mathcal{P}\left(\log \underline{\tilde{\boldsymbol{x}}}^{*}-k_{v} \mathcal{P}\left(\underline{\tilde{\boldsymbol{\xi}}}^{b}\right) \mathcal{P}\left(\underline{\tilde{\boldsymbol{\xi}}}^{b}\right)^{*}\right. \\
& -k_{p} \mathcal{D}(\log \underline{\tilde{\boldsymbol{x}}}) \mathcal{D}\left(\underline{\tilde{\boldsymbol{\xi}}}^{b}\right)^{*}-k_{v} \mathcal{D}\left(\underline{\tilde{\boldsymbol{\xi}}}^{b}\right) \mathcal{D}\left(\underline{\tilde{\boldsymbol{\xi}}}^{b}\right)^{*} \\
& \quad-k_{p} \mathcal{D}\left(\underline{\tilde{\boldsymbol{\xi}}}^{b}\right) \mathcal{D}(\log \underline{\tilde{\boldsymbol{x}}})^{*}-k_{v} \mathcal{D}\left(\underline{\tilde{\boldsymbol{\xi}}}^{b}\right) \mathcal{D}\left(\underline{\tilde{\boldsymbol{\xi}}}^{b}\right)^{*}
\end{aligned}
$$

which, using (3.11) and (4.28), results in

$$
\dot{V}_{\xi}=-2 k_{p} A_{2}-2 k_{v} V_{\xi}
$$

where

$$
A_{2} \triangleq\left\langle\mathcal{P}(\log \underline{\tilde{\boldsymbol{x}}}), \mathcal{P}\left(\underline{\tilde{\boldsymbol{\xi}}}^{b}\right)\right\rangle+\left\langle\mathcal{D}(\log \underline{\tilde{\boldsymbol{x}}}), \mathcal{D}\left(\underline{\tilde{\boldsymbol{\xi}}}^{b}\right)\right\rangle .
$$

Replacing (4.36) and (4.32) in (4.31), and choosing $k_{p}$ and $\bar{k}_{p}$ such that

$$
\dot{V}_{\xi}+\dot{V}_{\log }=-2 k_{v} V_{\xi}
$$

which implies

$$
-2 k_{p} A_{2}+2 \bar{k}_{p} A_{1}=0
$$

Hence, for $A_{2} \neq 0$ we obtain

$$
k_{p}=\bar{k}_{p}\left(\frac{A_{1}}{A_{2}}\right)
$$

Finally, from (4.31) and (4.38)

$$
\dot{V}=-2 k_{v} V_{\xi}
$$

which is negative semi-definite, because, although $V_{\xi}$ is positive definite, $\dot{V}=0$ when $\underline{\tilde{\boldsymbol{\xi}}}^{b}=0$ even when $V \neq 0$, as $V=V_{\log }+V_{\xi}$. This way, for $k_{v}>0$ the origin $\underline{\tilde{\boldsymbol{x}}}=1$ is a stable equilibrium point for the closed-loop error dynamics given by (4.11) under control law given by (4.14).

In order to prove that the equilibrium point is asymptotically stable lets rely on

\footnotetext{
${ }^{10}$ Notice that, since both $\underline{\tilde{\xi}}^{b}$ and $\log \underline{\tilde{x}}$ are pure dual quaternions, $\frac{d}{d t} \mathcal{P}\left(\underline{\tilde{\xi}}^{b}\right)^{*}=k_{p} \mathcal{P}(\log \underline{\tilde{x}})+k_{v} \mathcal{P}\left(\underline{\tilde{\xi}}^{b}\right)=$ $-k_{p} \mathcal{P}(\log \underline{\tilde{x}})^{*}-k_{v} \mathcal{P}\left(\underline{\tilde{\xi}}^{b}\right)^{*}$.
} 
Matrosov's Theorem (refer to Section 3.2.3). Defining the auxiliary function $W_{2}=\left\langle\mathcal{P}\left(\underline{\tilde{\xi}}^{b}\right), \mathcal{P}(\log \underline{\tilde{x}})\right\rangle+\left\langle\mathcal{D}\left(\underline{\tilde{\xi}}^{b}\right), \mathcal{D}(\log \underline{\tilde{x}})\right\rangle$ and taking its time derivative we obtain

$$
\begin{aligned}
\dot{W}_{2}=-\frac{1}{2}\left(\frac{d}{d t} \mathcal{P}\left(\underline{\tilde{\boldsymbol{\xi}}}^{b}\right) \mathcal{P}(\log \underline{\tilde{\boldsymbol{x}}})\right. & \left.+\mathcal{P}(\log \underline{\tilde{\boldsymbol{x}}}) \frac{d}{d t} \mathcal{P}\left(\underline{\tilde{\boldsymbol{\xi}}}^{b}\right)\right) \\
& -\frac{1}{2}\left(\frac{d}{d t} \mathcal{D}\left(\underline{\tilde{\boldsymbol{\xi}}}^{b}\right) \mathcal{D}(\log \underline{\tilde{\boldsymbol{x}}})+\mathcal{D}(\log \underline{\tilde{\boldsymbol{x}}}) \frac{d}{d t} \mathcal{D}\left(\underline{\tilde{\boldsymbol{\xi}}}^{b}\right)\right) .
\end{aligned}
$$

Since $\underline{\tilde{\boldsymbol{\xi}}}^{b}=0$, from $(4.15)$ we have

$$
\frac{d}{d t}\left(\underline{\tilde{\boldsymbol{\xi}}}^{b}\right)=-k_{p} \log \underline{\tilde{\boldsymbol{x}}}
$$

therefore

$$
\begin{aligned}
& \frac{d}{d t} \mathcal{P}\left(\underline{\tilde{\boldsymbol{\xi}}}^{b}\right)=-k_{p} \mathcal{P}(\log \underline{\tilde{\boldsymbol{x}}})=k_{p} \mathcal{P}(\log \underline{\tilde{\boldsymbol{x}}})^{*}, \\
& \frac{d}{d t} \mathcal{D}\left(\underline{\tilde{\boldsymbol{\xi}}}^{b}\right)=-k_{p} \mathcal{D}(\log \underline{\tilde{\boldsymbol{x}}})=k_{p} \mathcal{D}(\log \underline{\tilde{\boldsymbol{x}}})^{*} .
\end{aligned}
$$

Replacing (4.42) in (4.41), and using (3.6) we have

$$
\begin{aligned}
\dot{W}_{2} & =-\frac{1}{2}\left(2 k_{p}\|\mathcal{P}(\log \underline{\tilde{\boldsymbol{x}}})\|^{2}+2 k_{p}\|\mathcal{D}(\log \underline{\tilde{\boldsymbol{x}}})\|^{2}\right) \\
& =-k_{p} V_{\log } .
\end{aligned}
$$

Since the value of $k_{p}$ obtained from (4.39) is valid only when $A_{2} \neq 0$ (i.e., outside the set $\{\dot{V}=0\}$ or, equivalently, when $\left.\underline{\tilde{\xi}}^{b} \neq 0\right)$, an appropriate value for $k_{p}$ must also be choose inside the set $\{\dot{V}=0\}$ (i.e., when $A_{2}=0$ ); that is,

$$
k_{p}= \begin{cases}\bar{k}_{p}\left(\frac{A_{1}}{A_{2}}\right) & , A_{2} \neq 0 \\ \bar{k}_{p} & , \text { otherwise }\end{cases}
$$

where $\bar{k}_{p}$ is a positive real number. This way, from (4.43) we have

$$
\dot{W}_{2}=-\bar{k}_{p} V_{\log }
$$

Defining $\bar{\omega} \triangleq\left(\bar{k}_{p} / 2\right) V_{\log }$, which is positive definite, the condition of (3.17) is satisfied. Therefore, the equilibrium $\underline{\tilde{\boldsymbol{x}}}=1$ is globally asymptotically stable. 


\subsection{Classic linear kinematic control}

In order to compare the proposed approach to a classic linear kinematic controller, this section briefly reviews the classic linear kinematic control based on dual quaternion algebra introduced by Pham et al. (2010) with the error function introduced by Figueredo et al. (2013).

In the classic approach, the feasible control inputs are given by ${ }^{11}$

$$
\boldsymbol{u}_{f}=\boldsymbol{F} \boldsymbol{N}^{\dagger} \boldsymbol{K} \operatorname{vec}_{8}\left(1-\underline{\tilde{\boldsymbol{x}}}^{\prime}\right)
$$

where $\underline{\tilde{\boldsymbol{x}}}^{\prime}=\underline{\boldsymbol{x}}_{w b}^{*} \underline{\boldsymbol{x}}_{d}$ is the left invariant error, ${ }^{12} \boldsymbol{K}$ is a positive definite gain matrix and $\boldsymbol{F}$, which is given by (4.27), is the input mapping that guarantees feasible inputs for the nonholonomic mobile base. The matrix $\boldsymbol{N} \triangleq \overline{\boldsymbol{H}}_{8}\left(\underline{\boldsymbol{x}}_{d}\right) \boldsymbol{C}_{8} \boldsymbol{J}_{w b}$ is the constrained Jacobian (Figueredo et al., 2013), where $\overline{\boldsymbol{H}}_{8}(\cdot)$ is given by (3.16), $\boldsymbol{J}_{w b}$ is the whole-body Jacobian matrix given by (4.7), and $\boldsymbol{C}_{8} \triangleq \operatorname{diag}\left(\left[\begin{array}{llllllll}1 & -1 & -1 & -1 & 1 & -1 & -1 & -1\end{array}\right]\right)$.

As can be seen in (4.46), the control effort is proportional to the error. Therefore, when the error is too large the control signal will also be large, resulting in abrupt movements in the robot motion. This usually happens when the robot end-effector is far from the desired pose.

\subsection{Modified Jacobian Clamping}

Real robots present limits in the maximum values that their joints' angles may assume. Although such constraints were not considered during the controller design, a variation of the Jacobian Clamping presented by Colome and Torras (2015) (see Section 2.4) is proposed to be used with the nonlinear control. This strategy works by replacing (4.27) with

$$
\boldsymbol{u}_{f}=\boldsymbol{F} \boldsymbol{H}\left(\boldsymbol{J}_{w b} \boldsymbol{H}\right)^{\dagger} \operatorname{vec}_{8}\left(\frac{1}{2} \underline{\boldsymbol{x}}_{w b} \underline{\boldsymbol{u}}_{\xi}\right)
$$

where $\boldsymbol{H}$ is a diagonal matrix whose elements $h_{i}$ are defined as

$$
h_{i}= \begin{cases}0 & , \text { if }\left(q_{i}=q_{i_{\max }} \text { and sign } u_{i}=1\right) \text { or }\left(q_{i}=q_{i_{\min }} \text { and } \operatorname{sign} u_{i}=-1\right), \\ 1 \quad, \text { otherwise },\end{cases}
$$

\footnotetext{
${ }^{11}$ The actual classic kinematic controller is given by $\boldsymbol{u}=\boldsymbol{J}_{w b}^{\dagger} \boldsymbol{K} \mathrm{vec}_{8}\left(\underline{\boldsymbol{x}}_{d}-\underline{\boldsymbol{x}}\right)$, but it must adapted because of two main reasons: the error function is different from the one used in the present paper and the resulting control input $\boldsymbol{u}$ is not necessarily feasible.

${ }^{12}$ The error $\underline{\tilde{x}}^{\prime}$ differs from the one presented in (4.8), because it is expressed using the dual quaternions as presented in (3.7), but both expressions are equivalent.
} 
where $q_{i_{\max }}$ and $q_{i_{\min }}$ are the maximum and the minimum joint angles, respectively and $u_{i}$ is the control input given by (4.27). The modification of (2.3) proposed in (4.47) allows the joint to be unblocked if the control signal is such that the joint is being pushed away from its limit. This way, the robot does not have to permanently lose a degree of freedom if a joint is blocked at a given moment.

\subsection{Chapter Conclusions}

This chapter presented the whole-body modeling and nonlinear kinematic control of the mobile manipulator used in this work (Fig. 4.1). Section 4.1 showed the obtention of the whole-body kinematic model, through a systematic procedure using dual quaternion algebra. Section 4.2 presented the design of the nonlinear kinematic controller and the stability for the end-effector closed-loop error dynamics given by (4.11) under control law given by (4.14), through Lyapunov's Direct Method and Matrosov's Theorem. Section 4.3 reviewed the classic kinematic control strategy, which is used as base of evaluation for the nonlinear control scheme. Section 4.4 presented the proposed modification to the Jacobian Clamping, that potentially allows the robot's joint to, once blocked, not remain blocked during the rest of the experiment, allowing the robot to regain the lost degree of freedom. 


\section{5 Experiments and Results}

This chapter presents the experiments performed in order to evaluate the proposed control strategy. Section 5.1 presents the experimental setup utilized in this work and explains the task to be executed. Section 5.2 shows the obtained results and presents a discussion about them.

\subsection{Experimental Setup}

In order to assess the performance of the proposed approach, experiments were done using a five-DOF manipulator serially coupled to a nonholonomic mobile base, shown in Fig. 4.1. The implementation was done in Matlab 2016a and $\mathrm{C}++$ with the computational library DQ Robotics ${ }^{1}$ for dual quaternion algebra, using the operational system Ubuntu 14.04 LTS 64 bits with Robot Operating System (ROS Indigo). Unless stated otherwise, all tests were performed with the $\mathrm{C}++$ version of the code. All the real experiments were performed in a computer equipped with Intel Xeon i7 $2.4 \mathrm{GHz}$ octacore processor with 12 GB RAM and all the simulations were performed in a computer Intel Core i7-6500U CPU @ $2.50 \mathrm{GHz} \times 4,8 \mathrm{~GB}$ RAM. A Microsoft Kinect $^{\mathrm{TM}}$ sensor located at the ceiling and two fiducial markers were used in order to obtain the robot's end-effector pose and the pose of the goal. Unless stated otherwise, all simulations had the same stop condition, which consists of 50 consecutive iterations in which the error norm is smaller than $10^{-4}$, whereas for the all the real experiment, the stop criterion was an (Euclidean) error norm of less

\footnotetext{
${ }^{1}$ http://dqrobotics.sourceforge.net/
} 
than 0.04 (i.e., $\left.\left\|\operatorname{vec}_{8}(1-\underline{\tilde{x}})\right\|<0.04\right)$.

In order to compare the nonlinear controller to the linear controller a test scenario was designed, as shown in Fig. 5.1, where the goal was to move a small cube to a desired location and then drop it into a basket. Both controllers received only the desired end-effector pose and generated the corresponding joints control inputs that regulated the end-effector pose.

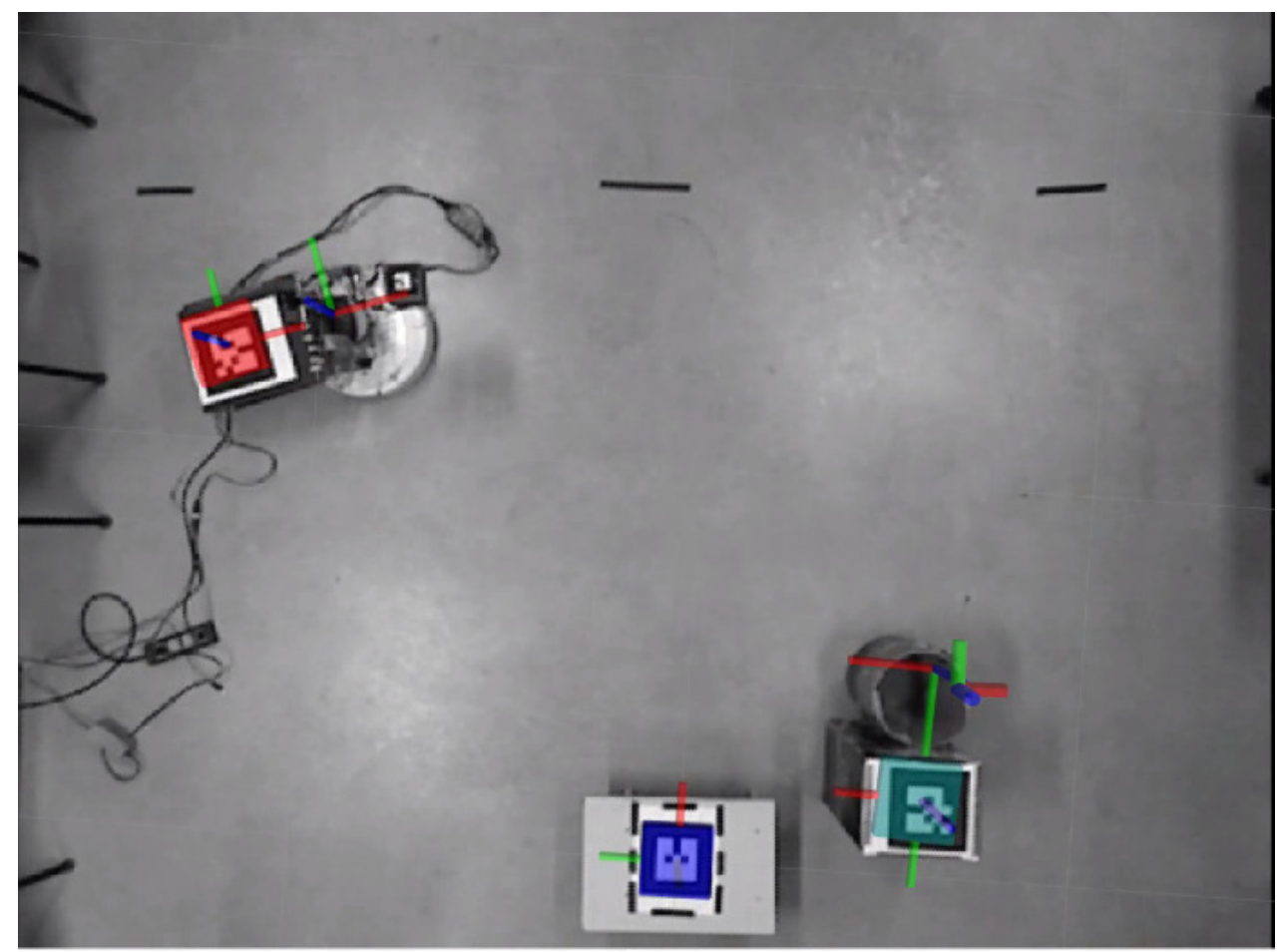

Figure 5.1: In the test scenario, the goal consisted of moving a small cube to a desired location and then dropping it into a basket. Both the robot's end-effector pose and the pose of the goal were measured in real time by using a Kinect sensor located at the ceiling and fiducial markers.

\subsection{Results}

Three groups of tests were performed: the first one was done in simulation to evaluate the effects of the gains $\bar{k}_{p}$ and $k_{v}$ on the controller behavior; the second one was done using the real robot to compare the proposed nonlinear strategy with the classic linear kinematic controller; and the last one was done both in simulation and using the real platform, in order to test the proposed technique to deal with joint constraints.

\subsubsection{Effects of the Nonlinear Controller Gains}

In the first set of tests, $\bar{k}_{p}=0.6$ and the values of $k_{v}$ were increased progressively. The coefficients of the dual quaternion that corresponds to the end-effector pose are shown in Fig. 5.2, where the reference pose is given by the green dotted line. The gain $k_{v}$ has an 
effect of attenuating the oscillatory behavior generated by the gain $\bar{k}_{p}$, allowing the robot to have smoother movements, but at the expense of a slower convergence rate.
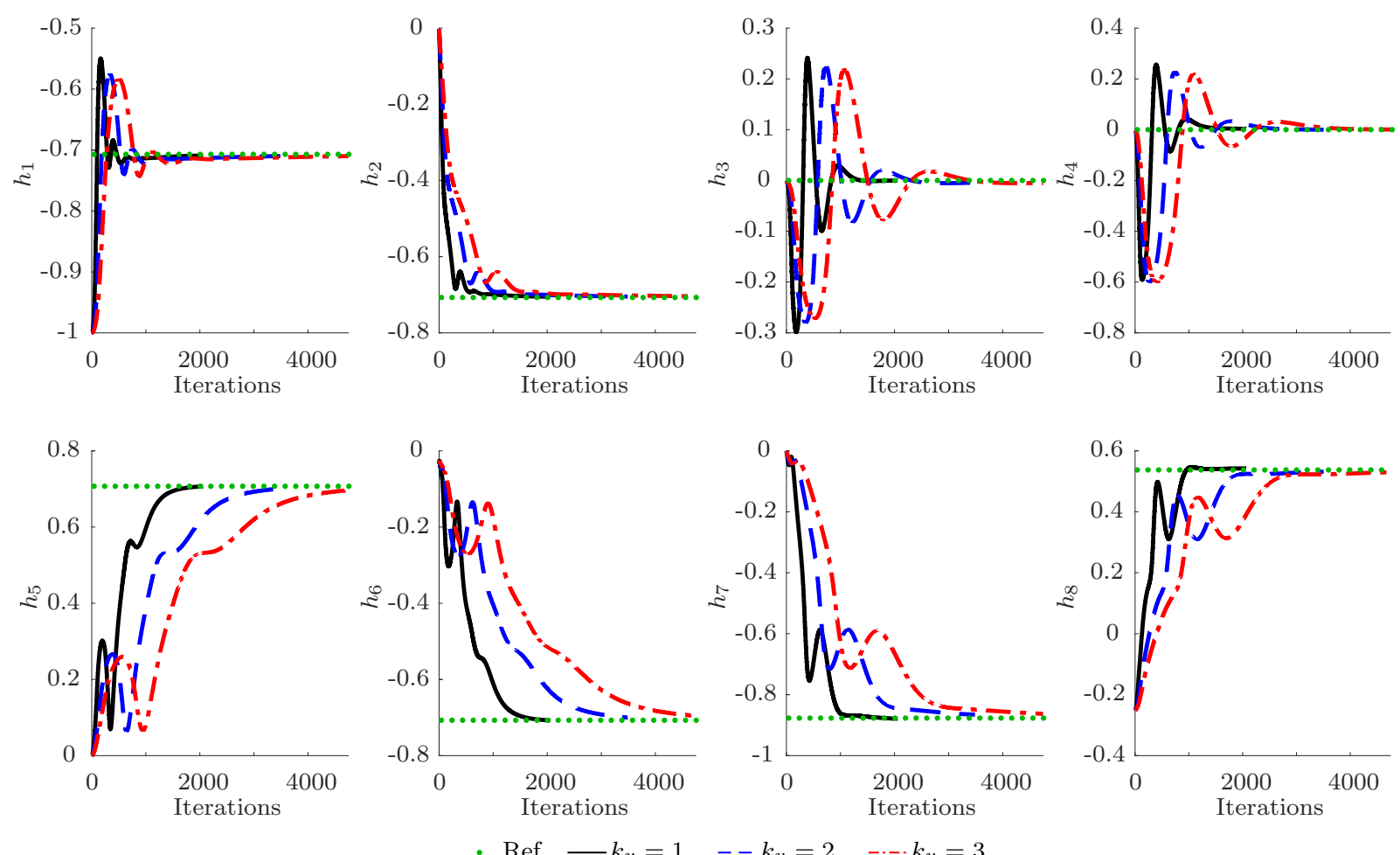

Figure 5.2: Coefficients of the dual quaternion corresponding to the end-effector pose. The dotted green curve corresponds to the desired pose and in all experiments $\bar{k}_{p}=0.6$. The solid black curve corresponds to $k_{v}=1$, the blue dashed curve corresponds to $k_{v}=2$, and the red dot-dashed curve corresponds to $k_{v}=3$.

In the second set of tests, the values of $\bar{k}_{p}$ were increased progressively while $k_{v}=2$. The coefficients of the dual quaternion that corresponds to the end-effector pose are shown in Fig. 5.3, where the reference pose is given by the green dotted line. As the gain $\bar{k}_{p}$ increases, the end-effector movements become more abrupt and oscillatory but the end-effector stabilizes faster.

In summary, $\bar{k}_{p}$ affects the convergence rate whereas $k_{v}$ attenuates the oscillatory behavior.

\subsubsection{Comparison of Controllers}

The proposed nonlinear strategy and the classic linear kinematic controller were tested on a real platform (see Fig. 4.1) using the experimental setup described in Section 5.1.

In the experiment (see Fig. 5.1) the desired pose was provided by the Kinect sensor located at the ceiling and the end-effector pose was continuously estimated by using the measured value of the mobile base pose, which was obtained by using a fiducial marker on top of the base, together with the measurements of the manipulator joints angles and the whole-body kinematic model (4.6). Both robot and basket were placed arbitrarily in the 

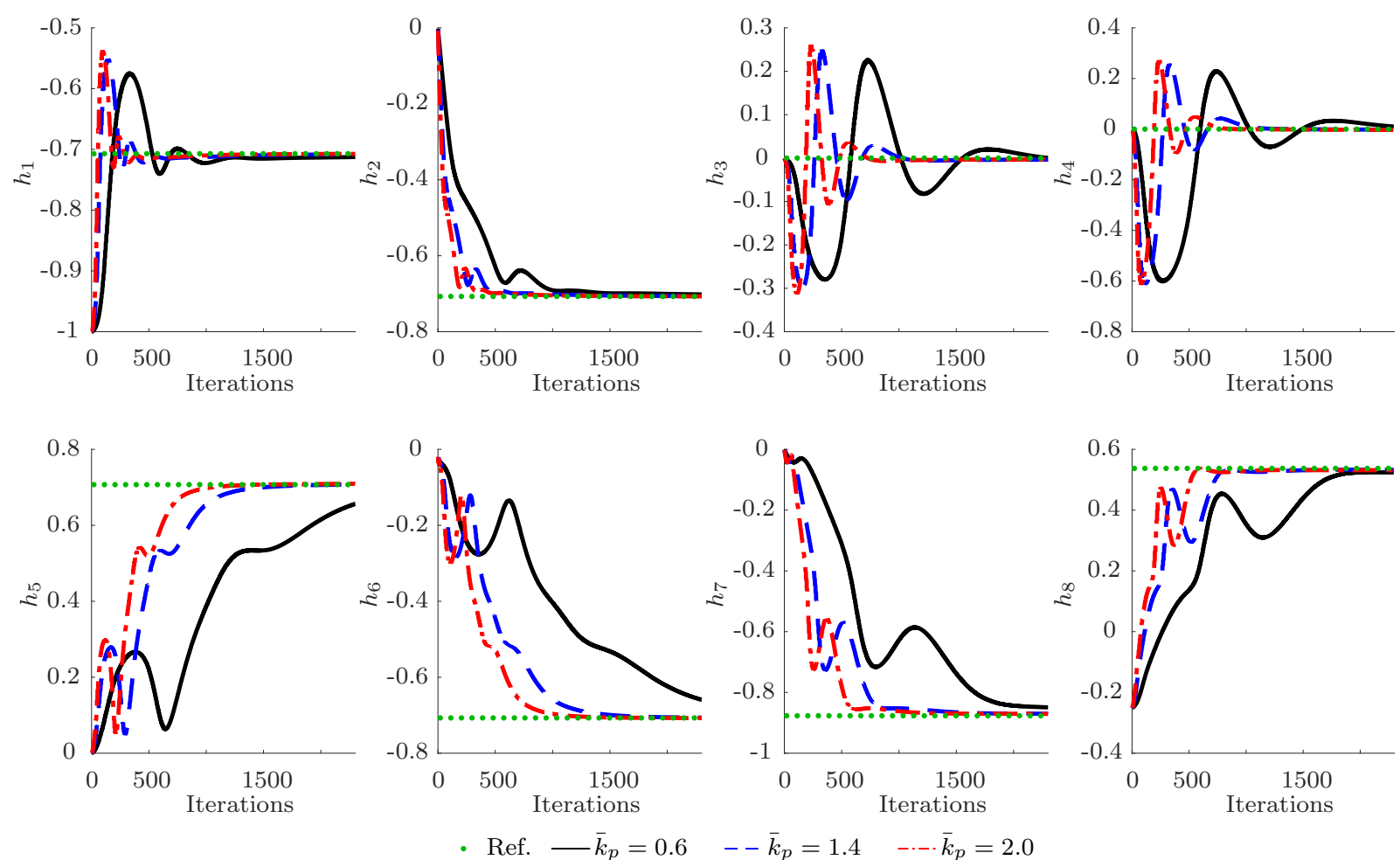

Figure 5.3: Coefficients of the dual quaternion corresponding to the end-effector pose. The dotted green curve corresponds to the desired pose and in all experiments $k_{v}=2$. The solid black curve corresponds to $\bar{k}_{p}=0.6$, the blue dashed curve corresponds to $\bar{k}_{p}=1.4$, and the red dot-dashed curve corresponds to $\bar{k}_{p}=2$.

workspace and both controllers gains were adjusted by trial and error in order to obtain similar convergence rates (around 650 iterations). More specifically, for the nonlinear controller $k_{v}=0.5, \bar{k}_{p}=0.6$ and $k_{p}$ was continuously calculated by using (4.39); for the classic linear controller $\boldsymbol{K}=0.5 \boldsymbol{I}$. Some snapshots of the experiment are shown in Fig. 5.4.

Fig. 5.5 shows the time response for all coefficients of the dual quaternion error (4.8). As expected, for all controllers the first coefficient converged to 1 and the others converged to zero, which implies that the end-effector pose converged to the desired one for both controllers. In addition, the nonlinear controller had similar convergence rate to the linear controller.

Fig. 5.6 presents a comparison between the norm of the end-effector pose error for both controllers. As expected, both controllers have approximately the same convergence rate as their parameters were chosen in order to obtain this behavior. However, Fig. 5.7 shows that the nonlinear controller generates smoother control inputs than the linear controller. This fact is also confirmed in Table 5.1, which presents the comparison between the control efforts of both controllers; indeed, the effort of the nonlinear controller is almost half than the effort of the linear controller.

The spikes present in the error curves of Fig. 5.5 and 5.6 are due to measurement errors. Indeed, although the pose information provided by Kinect is filtered before using it to estimate both the end-effector pose and the goal pose, the resultant measurements are 


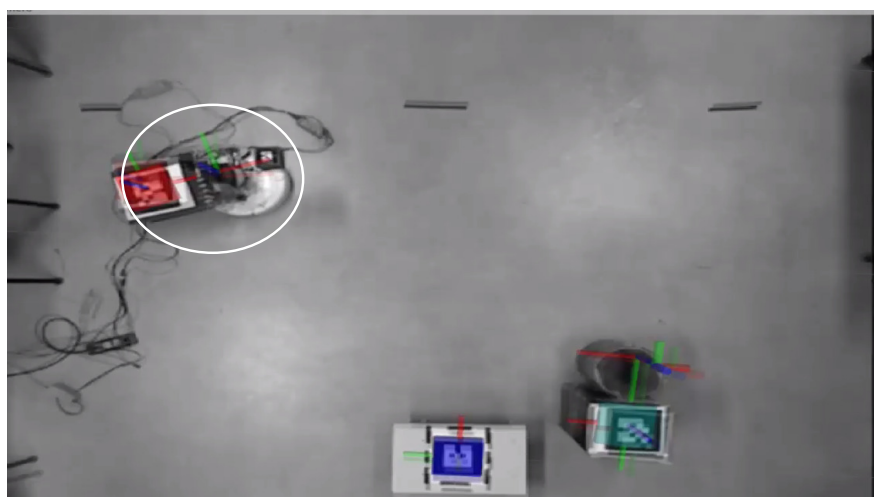

(a) The robot starts in the same configuration when both controllers are used.

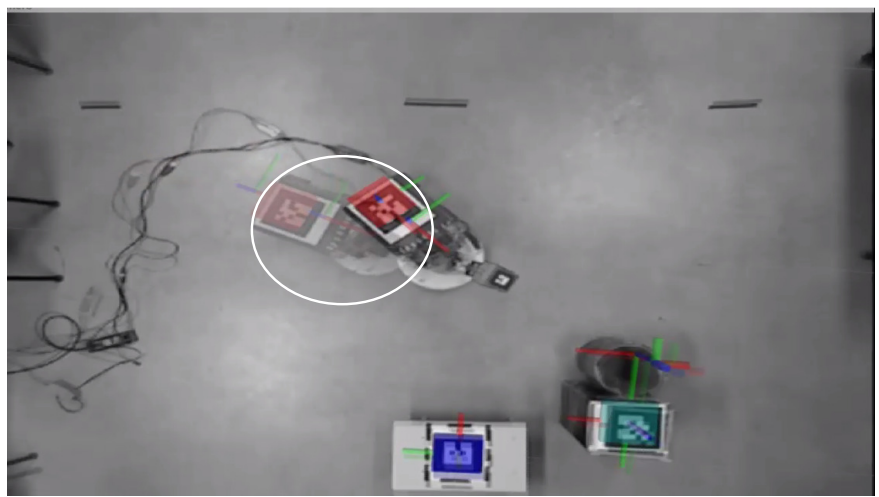

(b) In this intermediate snapshot the end-effector is closer to the desired pose when using the nonlinear controller than when the linear controller is used. This is due to the fact that the control inputs generated by the linear controller are proportional to the error, hence they are more abrupt at the beginning of the experiment, but become smoother as the end-effector converges to the desired pose.

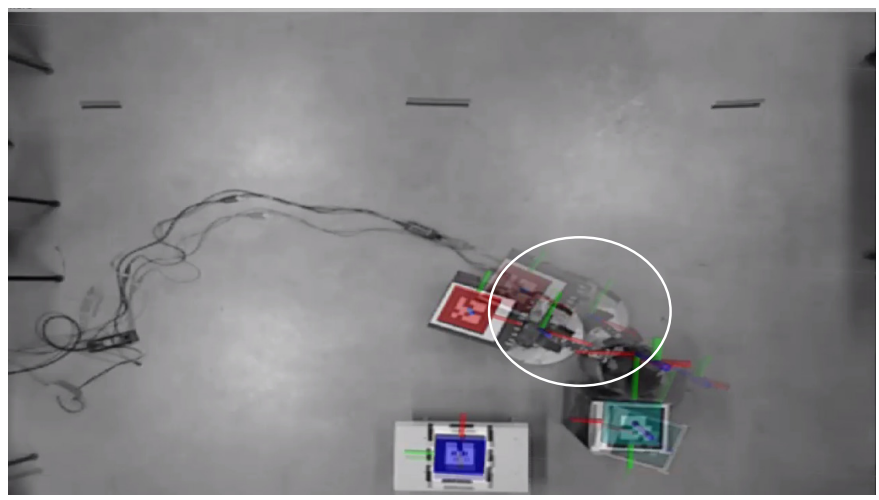

(c) The end-effector achieves the desired pose for both controllers, but the linear controller has an overshoot at the end of the trajectory.

Figure 5.4: Snapshots from the experiments, where the executed trajectories from both controllers are superimposed. The semi-transparent robot (marked with a white circle) shows the motion generated by the classic controller (4.46) and the regular (not transparent) robot shows motion generated by the nonlinear controller (4.27). 

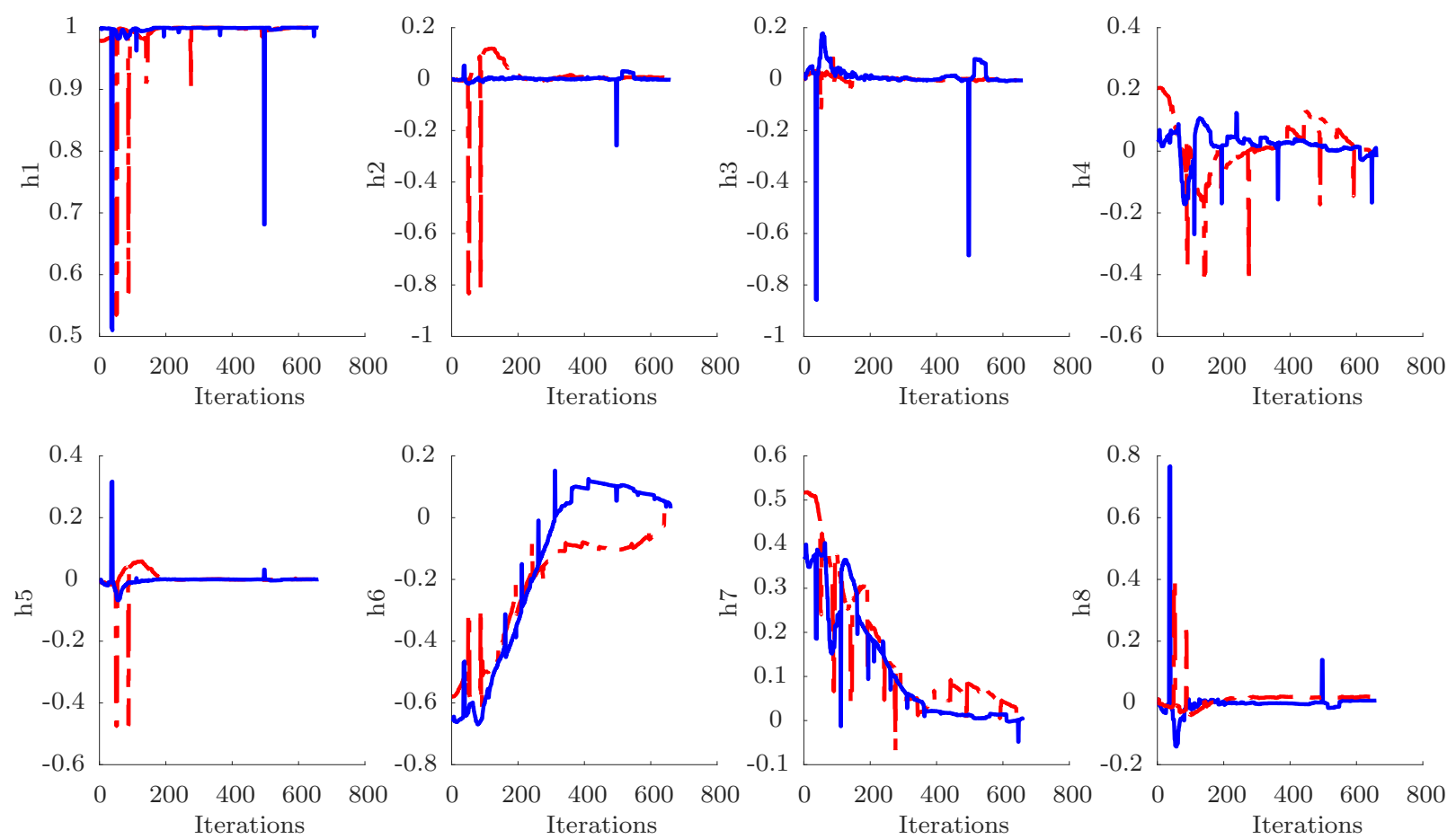

Figure 5.5: Coefficients of the dual quaternion error. The red dot-dashed curve corresponds to the nonlinear controller whereas the solid blue curve corresponds to the classic linear kinematic controller.

still noisy, which reflects on the calculated error.

\begin{tabular}{c|c|c}
\hline Metric & $\begin{array}{c}\text { Nonlinear } \\
\text { Controller }\end{array}$ & Linear Controller \\
\hline$\sqrt{\int_{0}^{\infty}\left\|\boldsymbol{u}_{f}(t)\right\|_{2}^{2} d t}$ & 4.8729 & 9.2067 \\
\hline
\end{tabular}

Table 5.1: Control Effort. Comparison of the control effort for all controllers

\subsubsection{Joints' Constraints}

Two experiments were performed in order to assess the efficiency of the modified Jacobian Clamping. The first one was done in simulation in Matlab, to verify the effects of joint's blockage in the convergence to poses that both violate and do not violate the joints' constraints, and the second one was performed in the real platform.

\subsubsection{Simulations}

Unless one desires that the mobile base remain restrict to a specified location, joint constraints apply only to the robotic arm. Therefore, the simulation was done, without any loss of generality, using only the manipulator. The implementation was done on Matlab 2016a with the computational library DQ Robotics. 


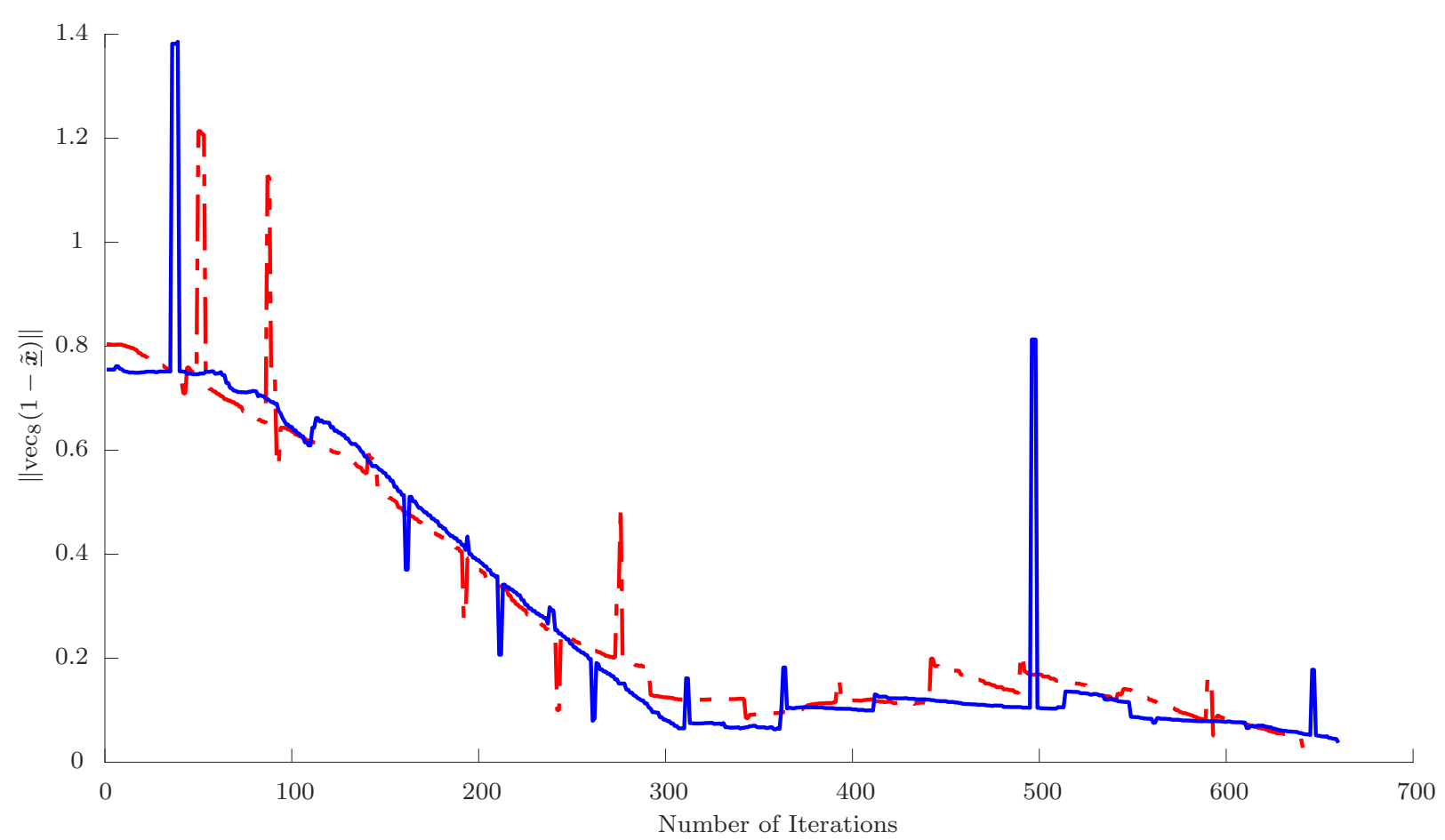

Figure 5.6: Euclidean norm the end-effector pose error $\left(\left\|\operatorname{vec}_{8}(1-\underline{\tilde{x}})\right\|\right)$. The red dot-dashed curve corresponds to the nonlinear controller whereas the solid blue curve corresponds to the classic linear kinematic controller.
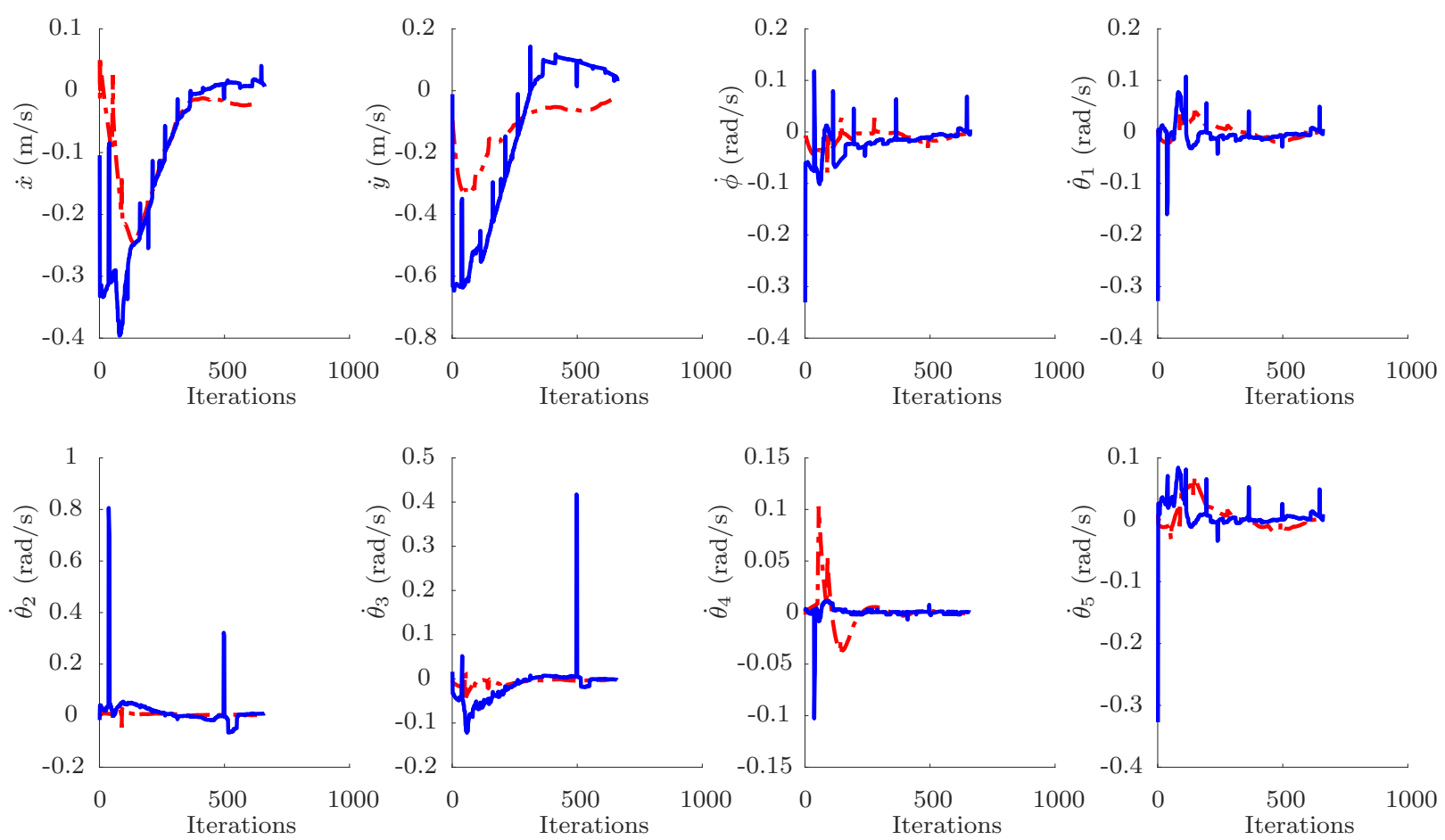

Figure 5.7: Control inputs (joints velocities). The red dot-dashed curve corresponds to the nonlinear controller whereas the solid blue curve corresponds to the classic linear kinematic controller. 
This technique was applied to the nonlinear controller. The desired end-effector pose was intentionally selected to be unfeasible without violating the joints' constraints. To evaluate the effects of this strategy when the desired pose is actually feasible within the joints' limits, the robot was ordered to go back to the initial pose as soon as it converged to the desired one. The robot's initial pose was given by $\underline{\boldsymbol{x}}_{0}=\boldsymbol{r}_{0}+\varepsilon(1 / 2) \boldsymbol{p}_{0} \boldsymbol{r}_{0}$, with $\boldsymbol{p}_{0}=\hat{\imath} 0.17+\hat{k} 0.34$ and $\boldsymbol{r}_{0}=\cos (3 \pi / 2)-\hat{k} \sin (3 \pi / 2)$ and the desired pose was given by $\underline{\boldsymbol{x}}_{d}=\boldsymbol{r}_{d}+\varepsilon(1 / 2) \boldsymbol{p}_{d} \boldsymbol{r}_{d}$, with $\boldsymbol{p}_{d}=\hat{\imath} 0.08+\hat{\jmath} 0.07+\hat{k} 0.33$ and $\boldsymbol{r}_{d}=\cos (1.4 \pi)-\boldsymbol{n}_{d} \sin (1.4 \pi)$, with $\boldsymbol{n}_{d}=\hat{\imath} 0.61-\hat{\jmath} 0.11-\hat{k} 0.78$, as shown in Fig. 5.8. The robot's joint angles were limited at $\theta_{i_{\max }}=\pi / 2$ and $\theta_{i_{\min }}=-\pi / 2$. The controller's gains are given by $k_{v}=2, \bar{k}_{p}=3$. The stop condition consisted of 200 consecutive iterations in which the error norm was smaller than $10^{-5}$.

Fig. 5.9 shows the time response for all coefficients corresponding to the dual quaternion end-effector pose. As expected, since the robot end-effector is unable to reach the desired pose without violating the joints' limits, it converged to the closest pose possible, in the sense of least squares ${ }^{2}$ (greyish area of the graph). However, when the desired end-effector pose is changed to be its initial pose and this pose is feasible, the robot asymptotically converges to it.

Fig. 5.10 shows the time response for the angles of the robot's joints. Without the use of Jacobian Clamping, the robot violates the joints' limits and converge to the desired pose, whereas when Jacobian Clamping is used the joints angles remain within its limits. It is worth noticing that, once blocked, the joints do not remain in this state for all the rest of the simulation, allowing the robot to regain the missing degree of freedom and properly converge to the initial pose.

\subsubsection{Experiments in the Real Robot}

The robot's motors are not very strong and depending on the robot's configuration they stop working, due to excess of torque needed to compensate the gravity. In this experiment, the modified Jacobian Clamping was used to limit the joints' angles (specially of the second and third joints) to avoid configurations that excessively force the robot's motors.

Two tests were performed, the first one without the usage of the modified Jacobian Clamping and the second one using it. For the second test, the robot's joint angles were limited with

$$
\begin{aligned}
\boldsymbol{q}_{\max } & =\left[\begin{array}{lllll}
\pi & -3 \pi / 8 & \pi / 2 & \pi / 2 & 3 \pi / 4
\end{array}\right]^{T}, \\
\boldsymbol{q}_{\min } & =\left[\begin{array}{lllll}
-\pi & -3 \pi / 4 & \pi / 4 & -\pi / 2 & -\pi / 2
\end{array}\right]^{T} .
\end{aligned}
$$

\footnotetext{
${ }^{2}$ Note that when no joint is violated, $\boldsymbol{H}=\boldsymbol{I}$, where $\boldsymbol{I}$ is the identity matrix. This results in $\boldsymbol{H}(\boldsymbol{J} \boldsymbol{H})^{\dagger}=\boldsymbol{J}^{\dagger}$. As $\boldsymbol{J}^{\dagger}$ provides the least squares solution, $\boldsymbol{H}(\boldsymbol{J} \boldsymbol{H})^{\dagger}$ also provides the least squares solution.
} 


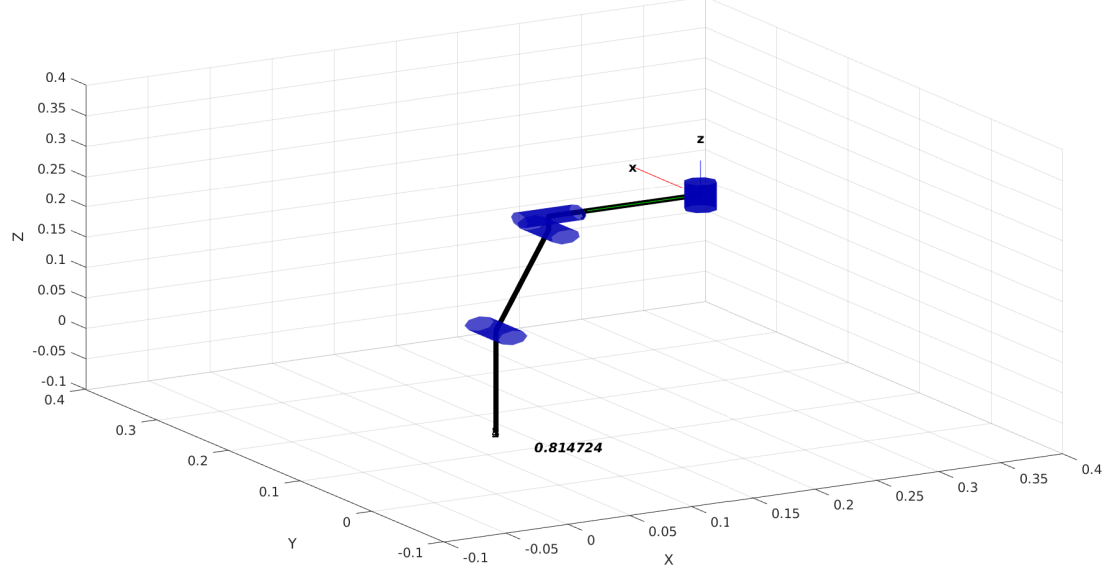

(a) Initial robot configuration.

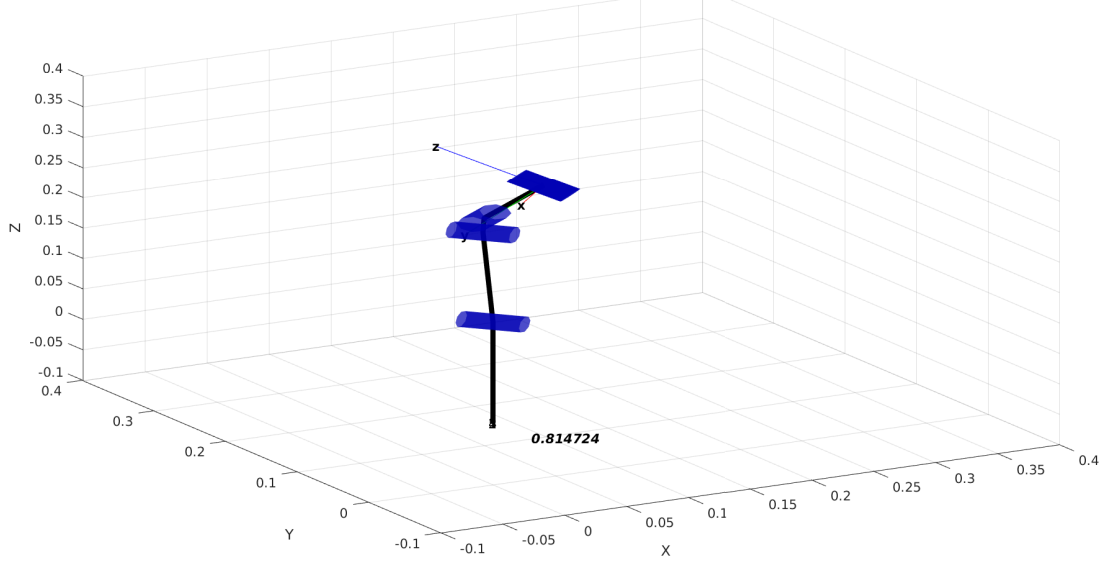

(b) Desired robot configuration. This configuration is unfeasible without violating the joints' constraints.

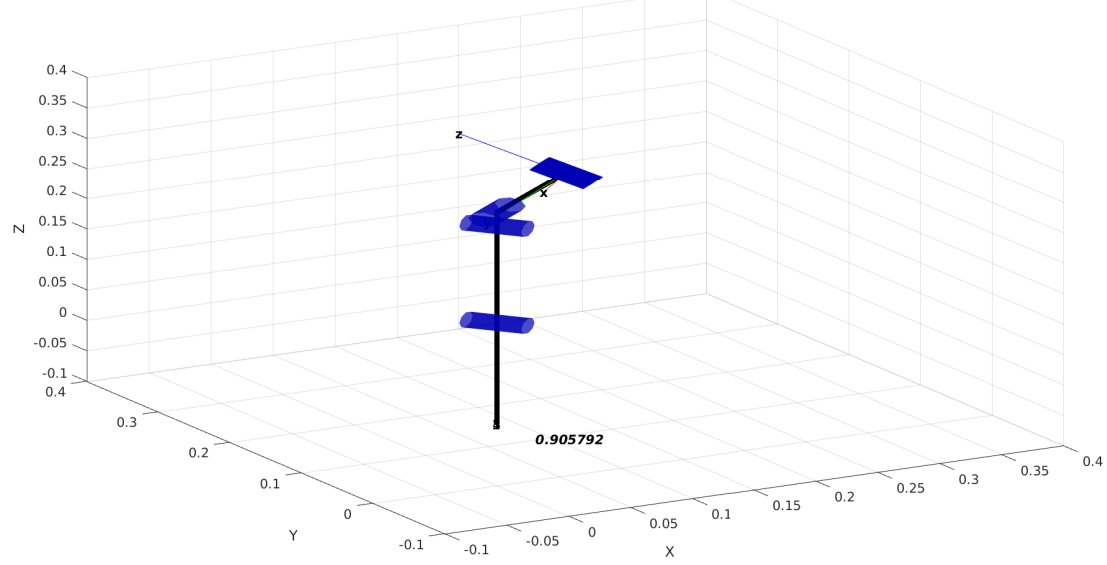

(c) Final robot configuration. As the desired end-effector pose is unreachable within the joints' constraints, the robot converges to the closest pose possible, in the sense of least squares.

Figure 5.8: Snapshots from the simulation of the Jacobian Clamping in Matlab. 

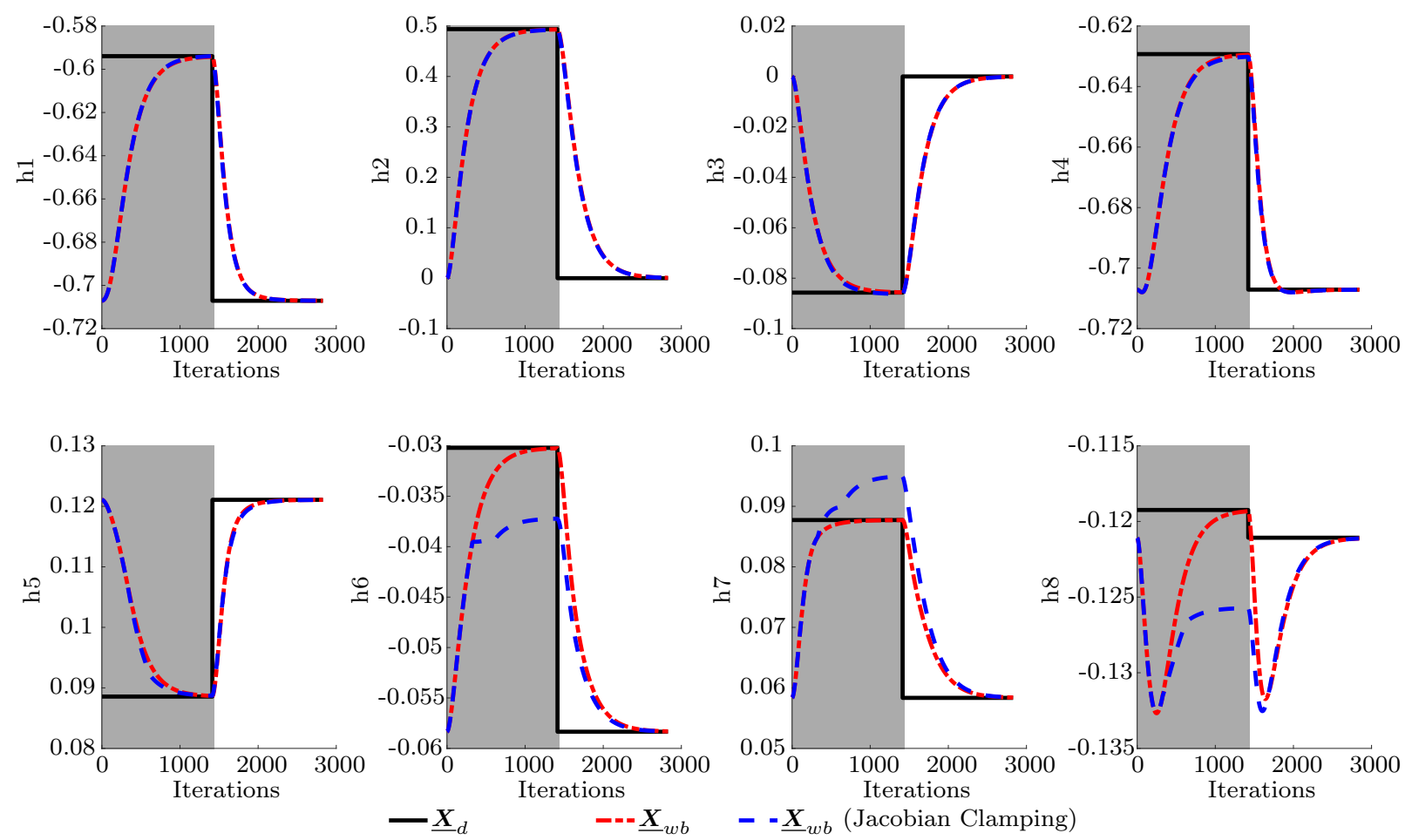

Figure 5.9: Coefficients of the dual quaternion corresponding to the end-effector pose. The greyish area of the graph indicates the phase in which the robot is trying to converge to a pose that is unreachable without violating the joints' limits. The solid black curve correspond to the desired pose of the nonlinear controller. The red dot-dashed curve corresponds to the nonlinear controller without the use of Jacobian Clamping whereas the dashed blue curve corresponds to the nonlinear controller with the use of Jacobian Clamping.
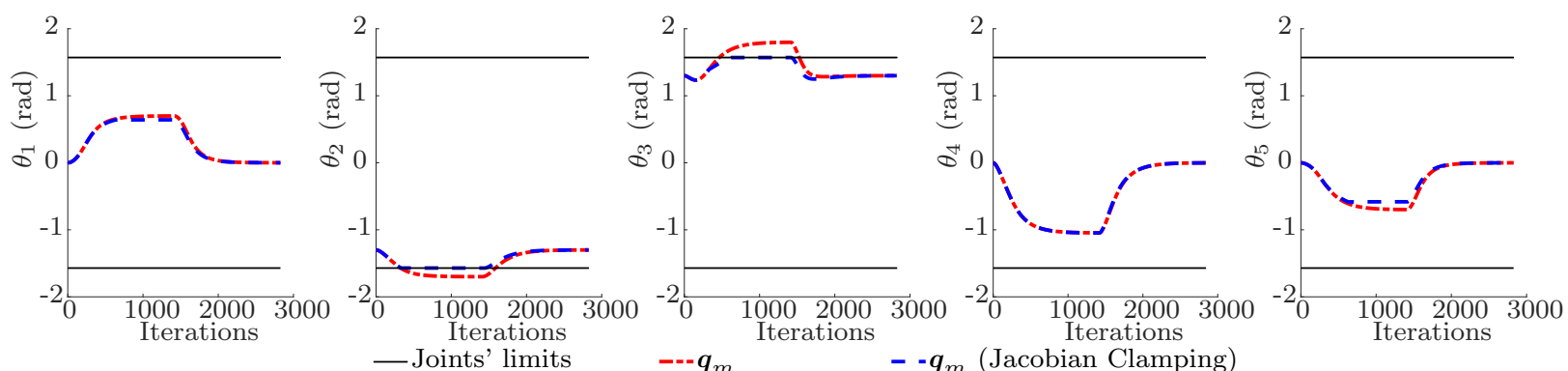

Figure 5.10: Joint angles. The solid black curve correspond to the joints' limits. The red dot-dashed curve corresponds to the nonlinear controller without the use of Jacobian Clamping whereas the dashed blue curve corresponds to the nonlinear controller with the use of Jacobian Clamping. 
Fig 5.11 shows snapshots from the experiment. For the test in which the modified Jacobian Clamping was not used, the motor from the third joint stopped working and, as a consequence, the robot was incapable of reaching the desired end-effector pose. However, when the proposed strategy to deal with joints' constraints is used, no motor is excessively forced and the robot is capable of converging to the desired end-effector pose.

Fig. 5.12 shows the time response for all coefficients of the dual quaternion that corresponds to the end-effector pose. As it can be noticed, in the test in which the modified Jacobian Clamping was not used, the robot is unable to converge to the desired end-effector pose, as a motor stopped working due to overheating.

Fig. 5.13 shows the time response for the angles of the robot's joints. It can be noticed that the modified Jacobian Clamping properly keeps the angles withing the desired limits whereas when this strategy is not used, some joints violate the limits and excessively stress the motors.

\subsection{Chapter Conclusions}

This chapter presented the experiments performed to assess the performance of the proposed control strategy. Section 5.1 presented the experimental setup used in this work, including a description of the external sensors used and the objects that the robot had to interact with. Section 5.2.1 showed the effects of the nonlinear controller's gains in its behavior, revealing that $\bar{k}_{p}$ affects its convergence rate whereas $k_{v}$ attenuates its oscillatory behavior. Section 5.2.2 presented the results obtained from the performed experiments, showing that the proposed control scheme is smother than the classic kinematic approach, while presenting smaller control effort and similar convergence rate. Section 5.2.3 presented the use of the proposed modification to the Jacobian Clamping to deal with joint constraints. Such technique does not affect the controller's convergence to a feasible pose and is able to ensure that, in the case that the pose is unreachable without violating the joints' constraints, the robot will converge to the nearest pose possible, in the sense of least squares. 

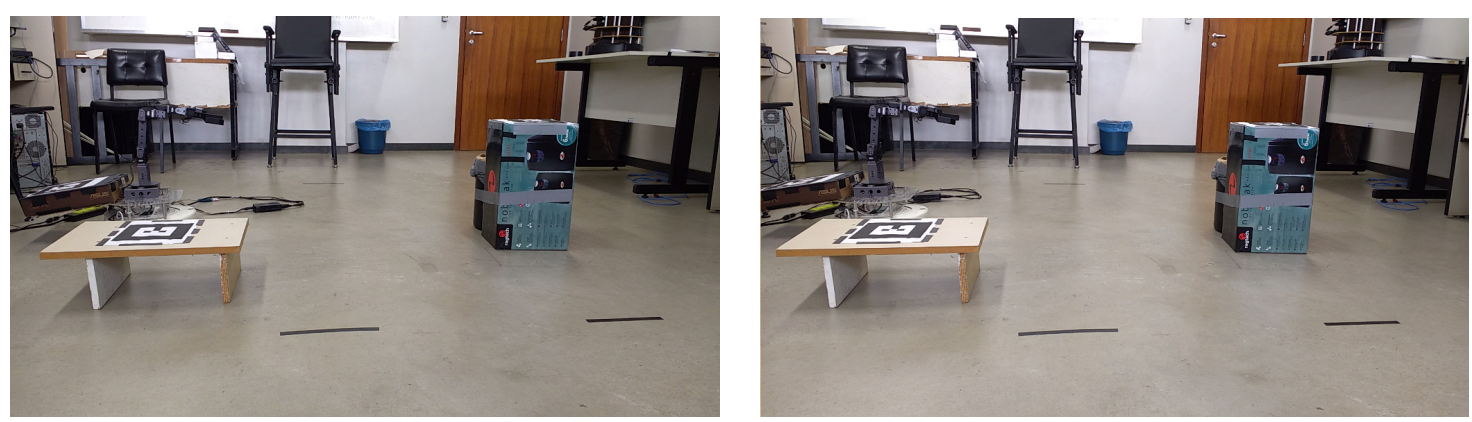

(a) The robot starts in the same configuration.
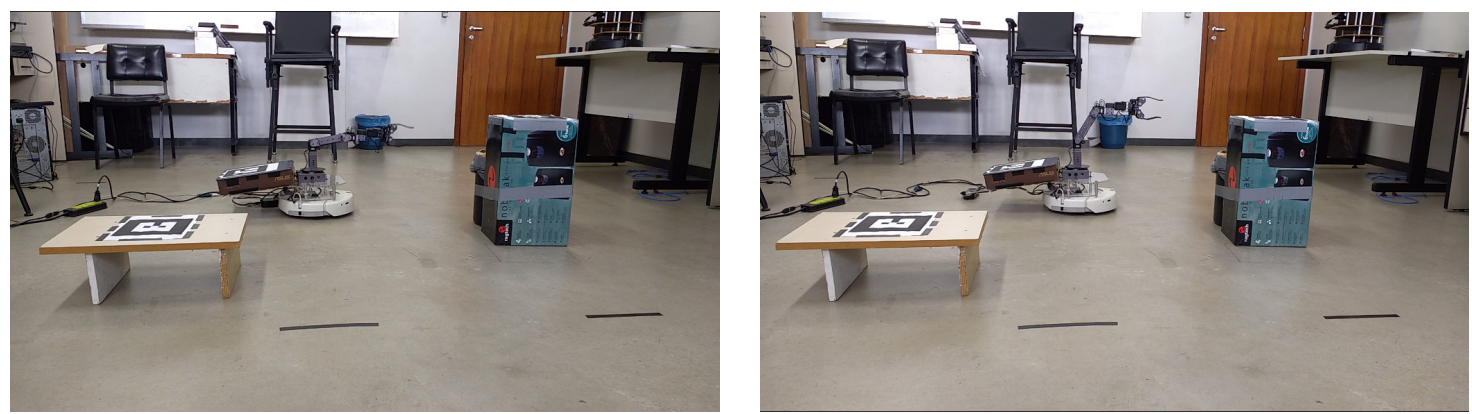

(b) In this intermediate snapshot the robot (left) that does not uses the modified Jacobian Clamping already violated its joints' limits and the robotic manipulator is almost fully extended.
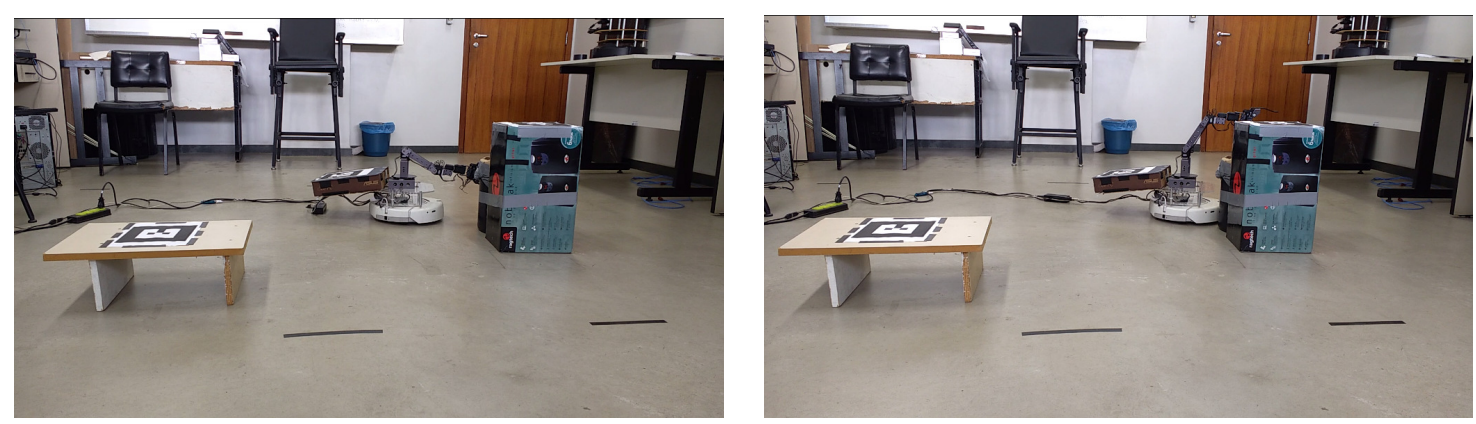

(c) In this final snapshot the motor from the third joint of the robot that does not uses the modified Jacobian Clamping stopped working due to the excess of torque. As a consequence, the robot is unable to converge to the desired end-effector pose, whereas the robot using the modified Jacobian Clamping (right) does not excessively force any motors and is able to properly stabilize the end-effector pose at the desired location.

Figure 5.11: Snapshots from the experiments, where the executed trajectories from both controllers are presented side by side. The images on the left show the motion generated by the nonlinear controller without the usage of the modified Jacobian Clamping and the images on the right show the motion generated by the nonlinear controller with modified Jacobian Clamping. 

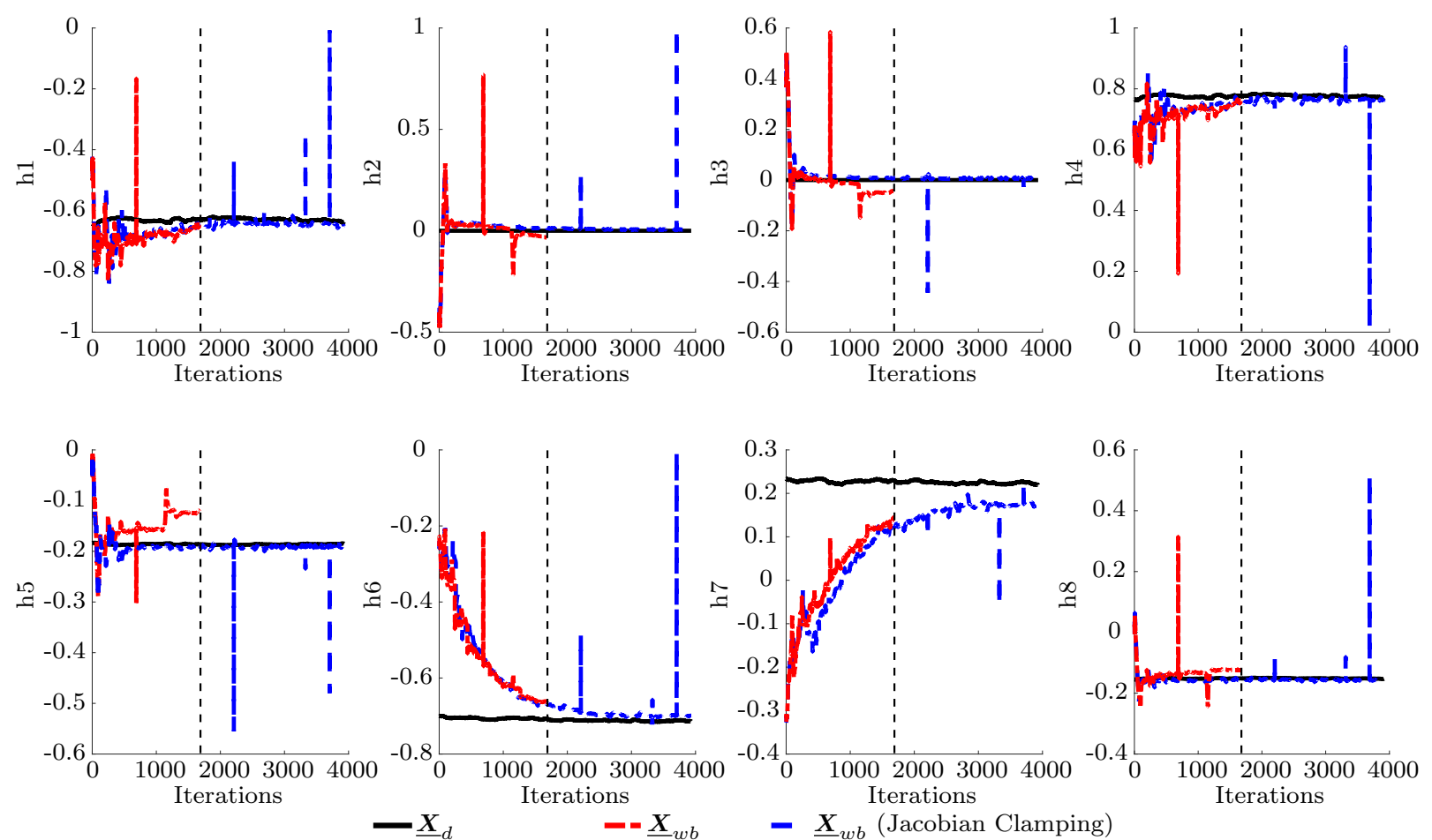

Figure 5.12: Coefficients of the dual quaternion corresponding to the end-effector pose. The vertical dashed black line indicates the moment in which the motor stopped working and the experiment was interrupted. The solid black curve correspond to the desired pose of the nonlinear controller. The red dot-dashed curve corresponds to the nonlinear controller without the use of Jacobian Clamping whereas the dashed blue curve corresponds to the nonlinear controller with the use of Jacobian Clamping.
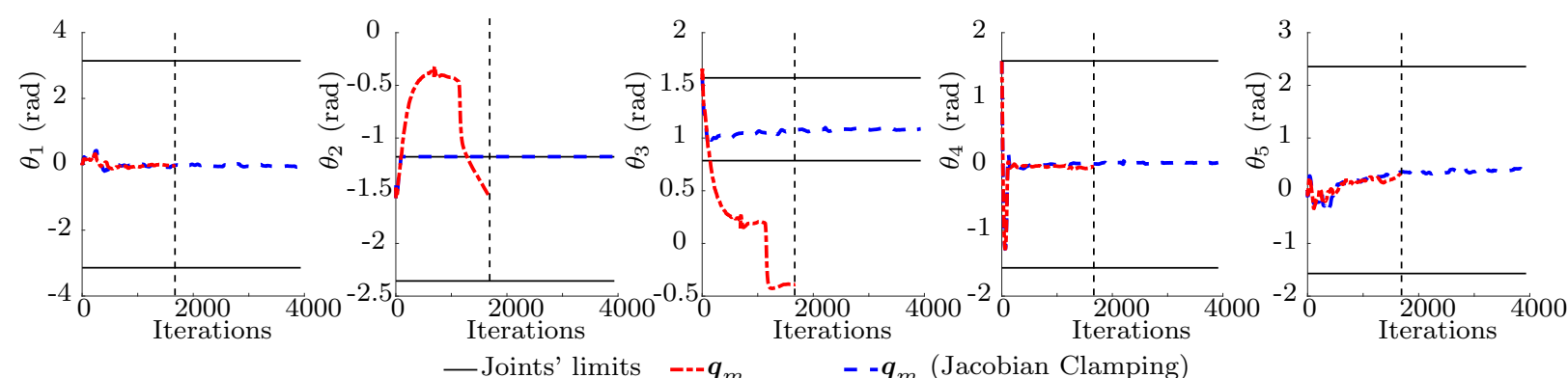

Figure 5.13: Joint angles. The solid black curve correspond to the joints' limits. The vertical dashed black line indicates the moment in which the motor stopped working and the experiment was interrupted. The red dot-dashed curve corresponds to the nonlinear controller without the use of Jacobian Clamping whereas the dashed blue curve corresponds to the nonlinear controller with the use of Jacobian Clamping. 


\section{6 \\ Conclusion and Future Works}

This master thesis presented a whole-body control strategy using feedback linearization and dual quaternion algebra, and applied the resultant technique to the motion control of a nonholonomic mobile manipulator. The nonlinear controller was adapted from the one proposed by Wang and $\mathrm{Yu}$ (2013) in order to use only the robot kinematics, therefore the resultant technique can be applied to velocity-actuated robots. The nonlinear pose controller was implemented in a nonholonomic mobile manipulator, which was modeling using the systematic procedure proposed by Adorno (2011). To deal with the nonholonomic constraints of the mobile base, the unfeasible input signals from the controller were remapped into feasible ones. Since the nonlinear controller acts as a dynamic trajectory generator, the robot movements are smoother compared to the ones generated by classic linear kinematic controllers.

The applicability of the proposed whole-body nonlinear controller was demonstrated by means of experimental results on a mobile manipulator composed of a five-DOF manipulator serially coupled to a nonholonomic mobile robot. The performance of the nonlinear controller was compared to the performance of a classic linear kinematic controller, showing that both have similar convergence rate but the nonlinear controller generates smoother movements while having smaller control effort. However, it is harder to tune the nonlinear controller's gains when compared to the classic controller, because the former has two parameters that influence each other whereas the latter has just one parameter directly related to the convergence rate.

Although the end-effector closed-loop dynamics is asymptotically stable, the whole- 
body asymptotic stability can be guaranteed only if the signal generated by the nonlinear controller lies in the range space of the whole-body Jacobian matrix. For the tracking problem this can be ensured by carefully generating a trajectory that belongs to the range space of the whole-body Jacobian matrix. However, this is not guaranteed for the regulation problem and future works will address this limitation. Furthermore, the controller design is done considering continuous time, whereas the implementation on the real robot is done in discrete time. The effects of such discretization will also be investigated in future works. Finally, although a modification in the Jacobian Clamping was proposed to deal with joints' constraints, alternatives to deal with joints' limits, as well as other hard constraints, will be explored in further works. 


\section{Bibliography}

Adorno, B. V. (2011). Two-arm Manipulation: From Manipulators to Enhanced HumanRobot Collaboration [Contribution à la manipulation à deux bras : des manipulateurs à la collaboration homme-robot]. PhD thesis.

Adorno, B. V. (2017). Robot Kinematic Modeling and Control Based on Dual Quaternion Algebra - Part I: Fundamentals.

Adorno, B. V., Fraisse, P., and Druon, S. (2010). Dual position control strategies using the cooperative dual task-space framework. In 2010 IEEE/RSJ International Conference on Intelligent Robots and Systems, pages 3955-3960. IEEE.

Alcocer, W., Vela, L., Blanco, A., Gonzales, J., and Oliver, M. (2012). Major Trends in the Development of Ankle Rehabilitation Devices. Dyna, 176:45-55.

Astolfi, A. and Praly, L. (2011). A LaSalle version of Matrosov theorem. In IEEE Conference on Decision and Control and European Control Conference, pages 320-324. IEEE.

Chan, T. F. and Dubey, R. V. (1995). A weighted least-norm solution based scheme for avoiding joint limits for redundant joint manipulators. IEEE Transactions on Robotics and Automation, 11(2):286-292.

Chen, T. L., Ciocarlie, M., Cousins, S., Grice, P. M., Hawkins, K., Kaijen Hsiao, Kemp, C. C., Chih-Hung King, Lazewatsky, D. A., Leeper, A. E., Hai Nguyen, Paepcke, A., Pantofaru, C., Smart, W. D., and Takayama, L. (2013). Robots for humanity: using assistive robotics to empower people with disabilities. IEEE Robotics $\&$ Automation Magazine, 20(1):30-39.

Chiaverini, S. (1997). Singularity-robust task-priority redundancy resolution for realtime kinematic control of robot manipulators. IEEE Transactions on Robotics and Automation, 13(3):398-410. 
Colome, A. and Torras, C. (2015). Closed-Loop Inverse Kinematics for Redundant Robots: Comparative Assessment and Two Enhancements. IEEE/ASME Transactions on Mechatronics, 20(2):944-955.

Dalibard, S., El Khoury, A., Lamiraux, F., Nakhaei, A., Taix, M., and Laumond, J.-P. (2013). Dynamic walking and whole-body motion planning for humanoid robots: an integrated approach. The International Journal of Robotics Research, 32(9-10):10891103.

de Almeida, M. M. and Raffo, G. V. (2015). Nonlinear Control of a TiltRotor UAV for Load Transportation. IFAC-PapersOnLine, 48(19):232-237.

De Luca, A. and Oriolo, G. (1991). The reduced gradient method for solving redundancy in robot arms. Robotersysteme, 7(May 1991):117-122.

De Luca, A., Oriolo, G., and Giordano, P. (2006). Kinematic modeling and redundancy resolution for nonholonomic mobile manipulators. In Proceedings 2006 IEEE International Conference on Robotics and Automation, 2006. ICRA 2006., volume 2006, pages 1867-1873. IEEE.

Dietrich, A., Wimbock, T., and Albu-Schaffer, A. (2011). Dynamic whole-body mobile manipulation with a torque controlled humanoid robot via impedance control laws. In 2011 IEEE/RSJ International Conference on Intelligent Robots and Systems, pages 3199-3206. IEEE.

Figueredo, L. F. C., Adorno, B. V., Ishihara, J. Y., and Borges, G. A. (2013). Robust kinematic control of manipulator robots using dual quaternion representation. In 2013 IEEE International Conference on Robotics and Automation, pages 1949-1955. IEEE.

Fonseca, M. d. P. A. and Adorno, B. V. (2016). Whole-Body Modeling and Hierarchical Control of a Humanoid Robot Based on Dual Quaternion Algebra. In 2016 XIII Latin American Robotics Symposium and IV Brazilian Robotics Symposium (LARS/SBR), pages 103-108. IEEE.

Gienger, M., Janben, H., and Goerick, C. (2006). Exploiting Task Intervals for Whole Body Robot Control. In 2006 IEEE/RSJ International Conference on Intelligent Robots and Systems, pages 2484-2490. IEEE.

Gilbert, E. G. and Ha, I. J. (1984). An approach to nonlinear feedback control with applications to robotics. IEEE Transactions on Systems, Man, and Cybernetics, SMC$14(6): 879-884$. 
Goncalves, V. M., Fraisse, P., Crosnier, A., and Adorno, B. V. (2016). Parsimonious Kinematic Control of Highly Redundant Robots. IEEE Robotics and Automation Letters, $1(1): 65-72$.

Hamilton, W. R. (1844). II. On quaternions; or on a new system of imaginaries in algebra. Philosophical Magazine Series 3, 25(163):10-13.

Han, D.-P., Wei, Q., and Li, Z.-X. (2008). Kinematic control of free rigid bodies using dual quaternions. International Journal of Automation and Computing, 5(3):319-324.

Ishida, T. (2004). Development of a small biped entertainment robot QRIO. In Micro-Nanomechatronics and Human Science, 2004 and The Fourth Symposium MicroNanomechatronics for Information-Based Society, 2004., pages 1-6. IEEE.

Jia, Y., Xi, N., and Nieves, E. (2014). Coordination of a nonholonomic mobile platform and an on-board manipulator. In 2014 IEEE International Conference on Robotics and Automation (ICRA), pages 4356-4361. IEEE.

Kussaba, H. T., Figueredo, L. F., Ishihara, J. Y., and Adorno, B. V. (2017). Hybrid kinematic control for rigid body pose stabilization using dual quaternions. Journal of the Franklin Institute, 354(7):2769-2787.

Lasota, P. A., Rossano, G. F., and Shah, J. A. (2014). Toward safe close-proximity human-robot interaction with standard industrial robots. In 2014 IEEE International Conference on Automation Science and Engineering (CASE), pages 339-344, Taipei, Taiwan. IEEE.

LaValle, S. M. (2006). Planning Algorithms. Cambridge University Press, Cambridge, first edition.

Li, Y. and Liu, Y. (2004). Control of a mobile modular manipulator moving on a slope. In Proceedings of the IEEE International Conference on Mechatronics, 2004. ICM '04., pages 135-140. IEEE.

Li, Y. and Liu, Y. (2005). Fuzzy logic self-motion planning and robust adaptive control for tip-over avoidance of redundant mobile modular manipulators. In Proceedings, 2005 IEEE/ASME International Conference on Advanced Intelligent Mechatronics., pages 1281-1286. IEEE.

Liégeois, A. (1977). Automatic Supervisory Control of the Configuration and Behavior of Multibody Mechanisms. IEEE Transactions on Systems, Man, and Cybernetics, $7(12): 868-871$. 
Liu, Y. and Li, Y. (2006). A New Method of Executing Multiple Auxiliary Tasks by Redundant Nonholonomic Mobile Manipulators. In 2006 IEEE/RSJ International Conference on Intelligent Robots and Systems, pages 1-6. IEEE.

Marinho, M. M., Figueredo, L. F. C., and Adorno, B. V. (2015). A dual quaternion linearquadratic optimal controller for trajectory tracking. In 2015 IEEE/RSJ International Conference on Intelligent Robots and Systems (IROS), pages 4047-4052. IEEE.

Matrosov, V. M. (1962). On the stability of motion. Journal of Applied Mathematics and Mechanics, 26(5):1337-1353.

Meeussen, W., Wise, M., Glaser, S., Chitta, S., McGann, C., Mihelich, P., Marder-Eppstein, E., Muja, M., Eruhimov, V., Foote, T., Hsu, J., Rusu, R. B., Marthi, B., Bradski, G., Konolige, K., Gerkey, B., and Berger, E. (2010). Autonomous door opening and plugging in with a personal robot. In 2010 IEEE International Conference on Robotics and Automation, pages 729-736. IEEE.

Mello, L. S., Raffo, G. V., and Adorno, B. V. (2016). Robust whole-body control of an unmanned aerial manipulator. In 2016 European Control Conference (ECC), pages 702-707. IEEE.

Nagasaka, K., Kawanami, Y., Shimizu, S., Kito, T., Tsuboi, T., Miyamoto, A., Fukushima, T., and Shimomura, H. (2010). Whole-body cooperative force control for a two-armed and two-wheeled mobile robot using Generalized Inverse Dynamics and Idealized Joint Units. In 2010 IEEE International Conference on Robotics and Automation, pages 3377-3383. IEEE.

Nishiwaki, K., Kagami, S., and Inoue, H. (2005). Object manipulation by hand using whole-body motion coordination. In IEEE International Conference Mechatronics and Automation, 2005, volume 4, pages 1778-1783. IEEE.

Oliveira, A. C. and Adorno, B. V. (2015). Balance Control of a Humanoid Robot Based on the Cooperative Dual Task-Space Framework. In XII Simpósio Brasileiro de Automação Inteligente (SBAI), pages 485-490.

Park, H. A. and Lee, C. S. G. (2013). Cooperative-Dual-Task-Space-based whole-body motion balancing for humanoid robots. In 2013 IEEE International Conference on Robotics and Automation, pages 4797-4802. IEEE.

Pham, H.-L., Perdereau, V., Adorno, B. V., and Fraisse, P. (2010). Position and orientation control of robot manipulators using dual quaternion feedback. In 2010 IEEE/RSJ International Conference on Intelligent Robots and Systems, volume pp., pages 658-663. IEEE. 
Quiroz-Omana, J. J. and Adorno, B. V. (2016). Parsimonious Kinematic Control of Nonholonomic Mobile Manipulators. In 2016 XIII Latin American Robotics Symposium and IV Brazilian Robotics Symposium (LARS/SBR), pages 73-78. IEEE.

Rego, B. S., Adorno, B. V., and Raffo, G. V. (2016). Formation Backstepping Control Based on the Cooperative Dual Task-Space Framework: A Case Study on Unmanned Aerial Vehicles. In 2016 XIII Latin American Robotics Symposium and IV Brazilian Robotics Symposium (LARS/SBR), pages 163-168. IEEE.

Salazar-Sangucho, F. R. (2014). Modelagem e Controle de Corpo Completo Usando Quatérnios Duais Aplicados a um Manipulador Móvel. Masters dissertation, Universidade Federal de Minas Gerais.

Salazar-Sangucho, F. R. and Adorno, B. V. (2014). Modelagem e Controle de Corpo Completo Usando Quatérnios Duais para um Manipulador Móvel. Congresso Brasileiro de Automática, pages 1-8.

Selig, J. M. (2005). Geometric Fundamentals of Robotics. Monographs in Computer Science. Springer New York, New York, NY.

Sentis, L. and Khatib, O. (2006). A whole-body control framework for humanoids operating in human environments. In Proceedings 2006 IEEE International Conference on Robotics and Automation, 2006. ICRA 2006., number May, pages 2641-2648. IEEE.

Siciliano, B., Sciavicco, L., Villani, L., and Oriolo, G. (2009). Robotics. Advanced Textbooks in Control and Signal Processing. Springer London, London.

Silva, F. F. A. and Adorno, B. V. (2016). Whole-Body Control of a Mobile Manipulator Using Feedback Linearization Based on Dual Quaternions. In 2016 XIII Latin American Robotics Symposium and IV Brazilian Robotics Symposium (LARS/SBR), pages 293-298, Recife. IEEE.

Slotine, J.-J. E. and Li, W. (1991). Applied Nonlinear Control. Prentice Hall.

Spong, M. and Vidyasagar, M. (1985). Roboust nonlinear control of robot manipulators. In 1985 24th IEEE Conference on Decision and Control, number December, pages 1767-1772. IEEE.

Vidyasagar, M. (1985). Control System Synthesis: A Factorization Approach. M. I. T. Press, Cambridge, MA.

Wang, X. and Yu, C. (2013). Unit dual quaternion-based feedback linearization tracking problem for attitude and position dynamics. Systems and Control Letters, 62(3):225-233. 
Wen, J. T.-Y. J.-Y. J. T.-Y., Delgado, K. K., and Kreutz-Delgado, K. (1991). The attitude control problem. Automatic Control, IEEE Transactions, 36(10):1148-1162.

Zghal, H., Dubey, R. V., and Euler, J. A. (1990). Efficient gradient projection optimization for manipulators with multiple degrees of redundancy. In Proceedings., IEEE International Conference on Robotics and Automation, pages 1006-1011, Cincinnati. IEEE Comput. Soc. Press.

Zhang, H., Jia, Y., and Xi, N. (2012). Sensor-based redundancy resolution for a nonholonomic mobile manipulator. In 2012 IEEE/RSJ International Conference on Intelligent Robots and Systems, pages 5327-5332. IEEE. 